\title{
Zinc Triggered Release of Encapsulated Cargo from Liposomes via a Synthetic Lipid Switch
}

Ruhani Sagar, Jinchao Lou, Alexa J. Watson, and Michael D. Best*

Department of Chemistry, University of Tennessee, 1420 Circle Drive, Knoxville, TN, 37996, United States

* Michael D. Best- orcid.org/0000-0001-8737-5910; Email: mdbest@utk.edu

\section{Table of Contents}

Supplemental Figures and Tables .................................... S2 - S16

Compound Spectra for Characterization Data .................. S16 - S36 


\begin{tabular}{|c|c|c|c|c|c|c|}
\hline \multicolumn{7}{|c|}{ Formulations explored for hydrophobic triggered release } \\
\hline Entry & Comments & $\begin{array}{c}\% \\
\text { ZRL }\end{array}$ & \% egg-PC & $\begin{array}{c}\% \\
\text { DOPE }\end{array}$ & $\begin{array}{c}\% \\
\text { PA }\end{array}$ & Outcome \\
\hline 1 & \multirow{3}{*}{$\begin{array}{l}\text { ZRL/PC based } \\
\text { liposomes }\end{array}$} & 20 & 80 & -- & -- & $\begin{array}{l}\text { Stable liposomes } \\
\text { minimal release }\end{array}$ \\
\hline 2 & & 30 & 70 & -- & -- & $\begin{array}{l}\text { Stable liposomes } \\
\text { minimal release }\end{array}$ \\
\hline 3 & & 40 & 60 & -- & -- & $\begin{array}{l}\text { Did not produce stable } \\
\text { liposomes }\end{array}$ \\
\hline 5 & \multirow{2}{*}{$\begin{array}{l}\text { ZRL/PC/DOPE based } \\
\text { liposomes } \\
\text { Fix ZRL } 25 \% \text { and vary } \\
\text { DOPE and PC }\end{array}$} & 25 & 35 & 40 & -- & $\begin{array}{l}\text { Did not produce stable } \\
\text { liposomes }\end{array}$ \\
\hline 6 & & 25 & 45 & 30 & & $\sim 15 \%$ release over 5 hours \\
\hline 7 & \multirow{3}{*}{$\begin{array}{l}\text { ZRL/PC/DOPE based } \\
\text { liposomes } \\
\text { Fix ZRL } 20 \% \text { and vary } \\
\text { DOPE and PC }\end{array}$} & 20 & 70 & 10 & -- & $\begin{array}{l}\text { Stable liposomes } \\
\text { minimal release }\end{array}$ \\
\hline 8 & & 20 & 60 & 20 & -- & $\begin{array}{l}\text { Stable liposomes } \\
\text { minimal release }\end{array}$ \\
\hline 9 & & 20 & 55 & 25 & -- & $\begin{array}{l}\text { Stable liposomes } \\
\text { minimal release }\end{array}$ \\
\hline 10 & \multirow{2}{*}{$\begin{array}{c}\text { Fix } 20 \% \text { ZRL and } 5 \% \\
\text { PA } \\
\text { Varying DOPE and PC }\end{array}$} & 20 & 65 & 10 & 5 & $\begin{array}{l}\text { Stable liposomes } \\
\text { minimal release }\end{array}$ \\
\hline 11 & & 20 & 45 & 30 & 5 & $\begin{array}{l}\text { Stable liposomes } \\
\text { minimal release }\end{array}$ \\
\hline 12 & \multirow{3}{*}{$\begin{array}{c}\text { Fix } 25 \% \text { ZRL and } 10 \% \\
\text { PA } \\
\text { Varying DOPE and PC }\end{array}$} & 25 & 45 & 20 & 10 & $\begin{array}{l}\text { Stable liposomes } \\
\text { minimal release }\end{array}$ \\
\hline 13 & & 25 & 40 & 25 & 10 & $\begin{array}{l}\text { Stable liposomes } \\
\text { minimal release }\end{array}$ \\
\hline 14 & & 25 & 35 & 30 & 10 & $\begin{array}{l}\text { Stable liposomes } \\
\text { minimal release }\end{array}$ \\
\hline 15 & \multirow{3}{*}{$\begin{array}{c}\text { Fix } 30 \% \text { ZRL and } 10 \% \\
\text { PA } \\
\text { Varying DOPE and PC }\end{array}$} & 30 & 30 & 30 & 10 & $\begin{array}{l}\text { Stable liposomes } \\
\text { minimal release }\end{array}$ \\
\hline 16 & & 30 & 20 & 40 & 10 & 20\% release over 2 hours \\
\hline 17 & & 30 & 10 & 50 & 10 & 85\% release over 8 hours \\
\hline
\end{tabular}

Table S1. Summary of formulations explored for hydrophobic triggered release using ZRL 1a containing different percentages of PC, DOPE, and PA. 


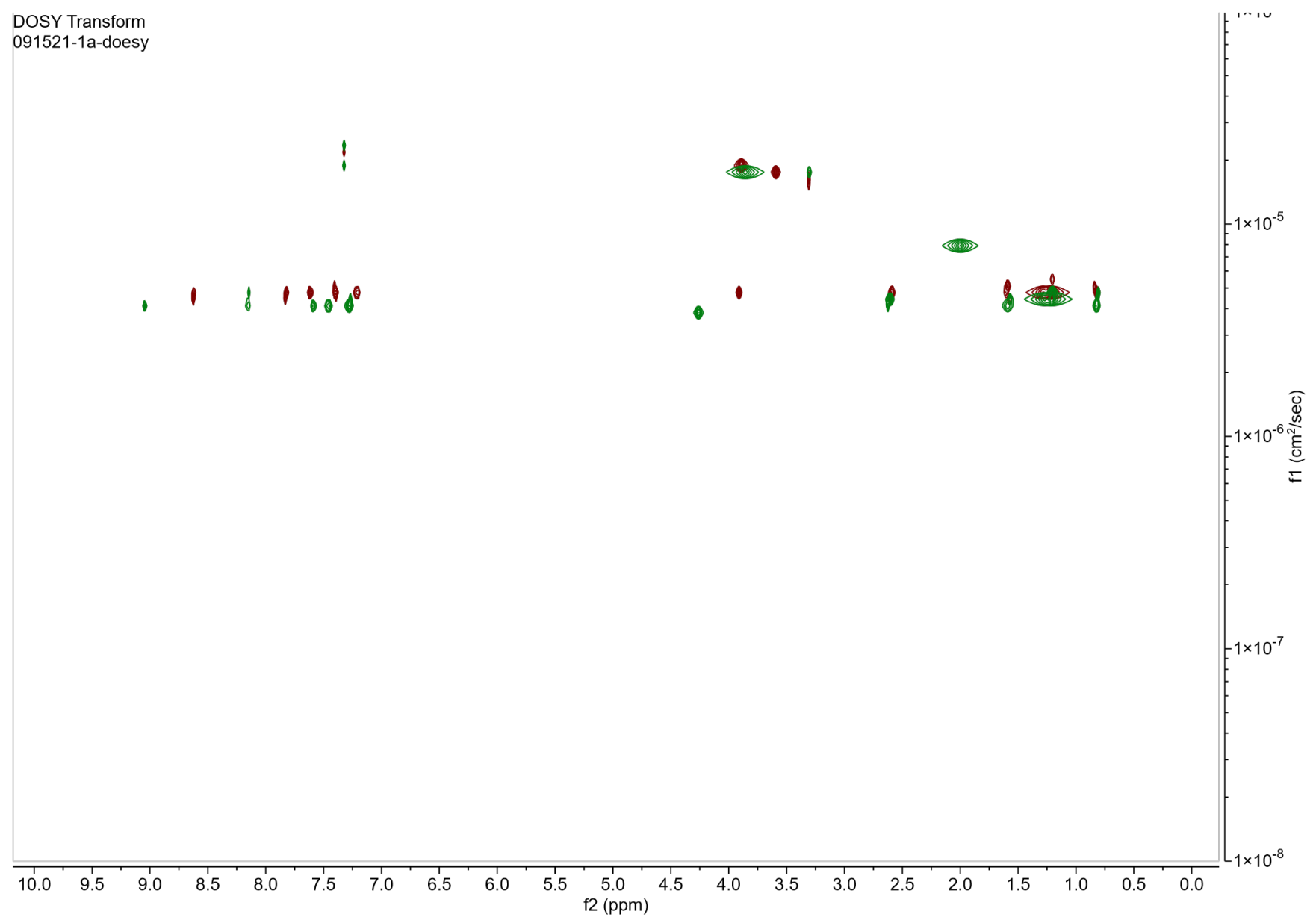

Figure S1. Representative diffusion ordered spectroscopy (DOSY) NMR spectra of $1 \mathrm{mM}$ of 1a in the absence (red) and presence (green) of $10 \mathrm{mM}$ zinc. The diffusion constant for 1a was found to be $5.57 \mathrm{e}-06$ for $1 \mathrm{a}$ and $4.6565 \mathrm{e}-$ 06 for $1 \mathrm{a}+10 \mathrm{mM} \mathrm{Zn}^{2+}$, averaged over three trials. 


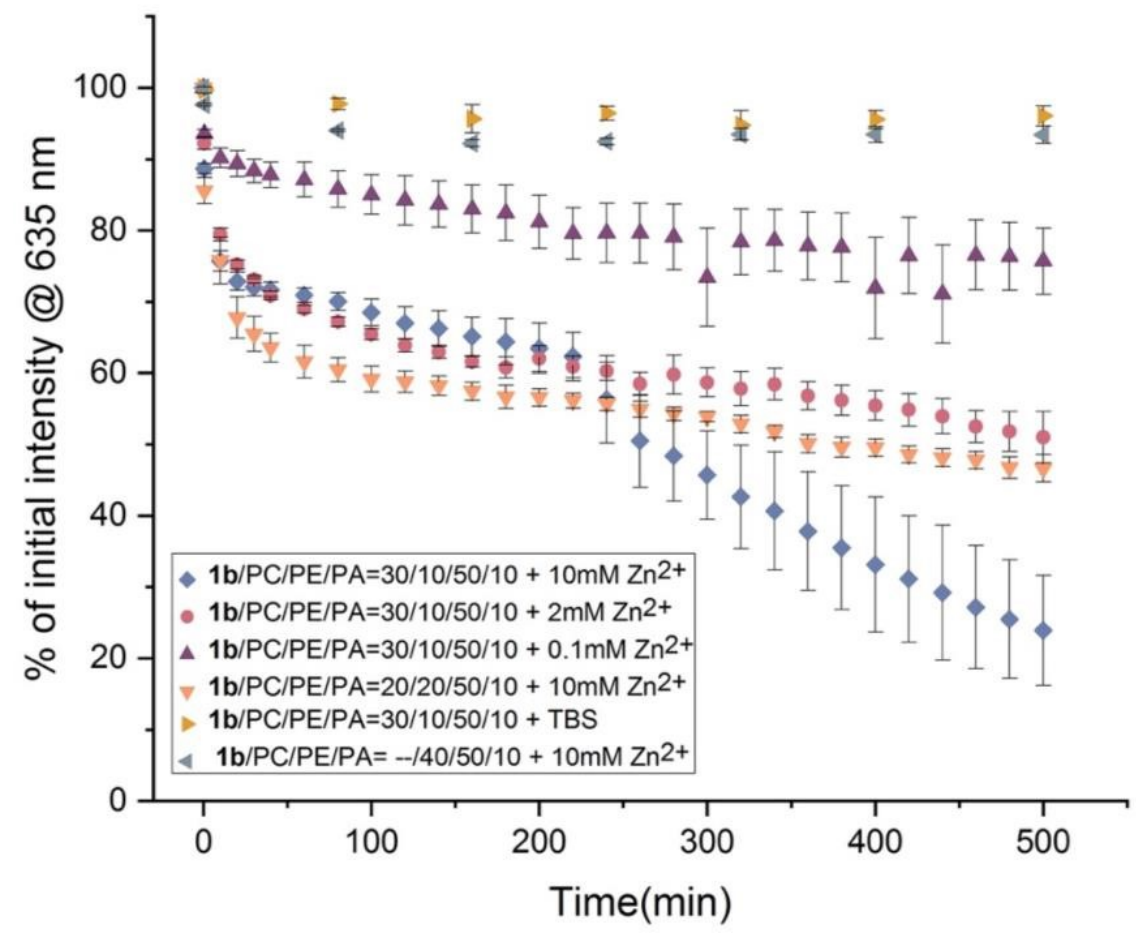

Figure S2. NR release profile over time for ZRL 1 b at 0-30\% of liposome composition containing 50\% DOPE, 10\% $\mathrm{PA}$ and $\mathrm{PC}$ as the remaining percentage. Error bars indicate standard error over triplicate experiments.

A

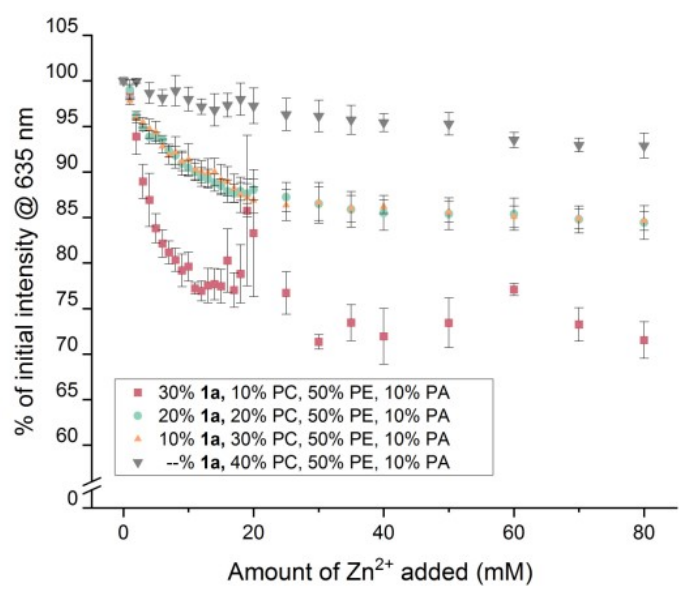

B

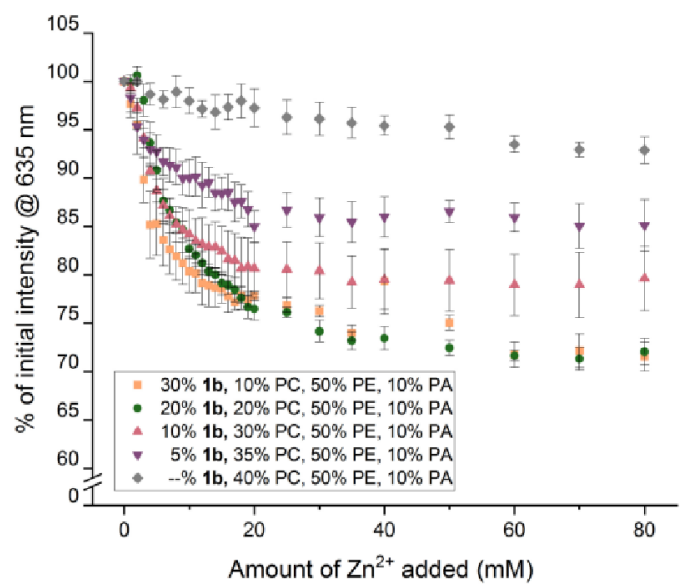

Figure S3. Results from titration experiments of NR liposomes containing ZRLs (A) 1a (B) 1b. Error bars indicate standard error over triplicate experiments. 


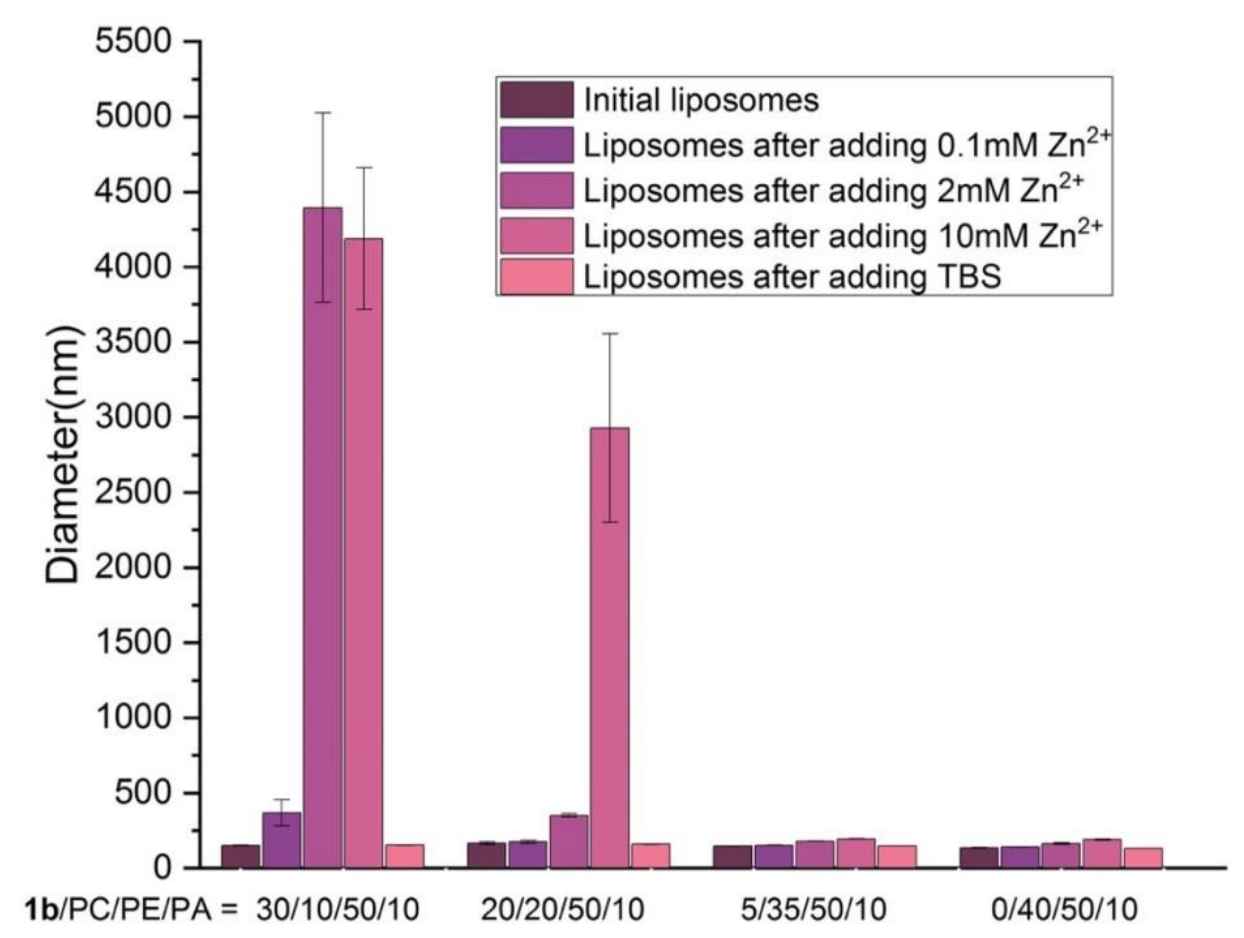

Figure S4. DLS analysis of liposomes containing 0-30\% $\mathbf{1 b}$ upon addition of varying zinc acetate concentrations. Dose-dependent increases in particle sizes were generally observed, with controls showing that incorporation of sufficient $\mathbf{1 b}$ is required for effects to be observed. Error bars indicate standard error over triplicate experiments. 
A
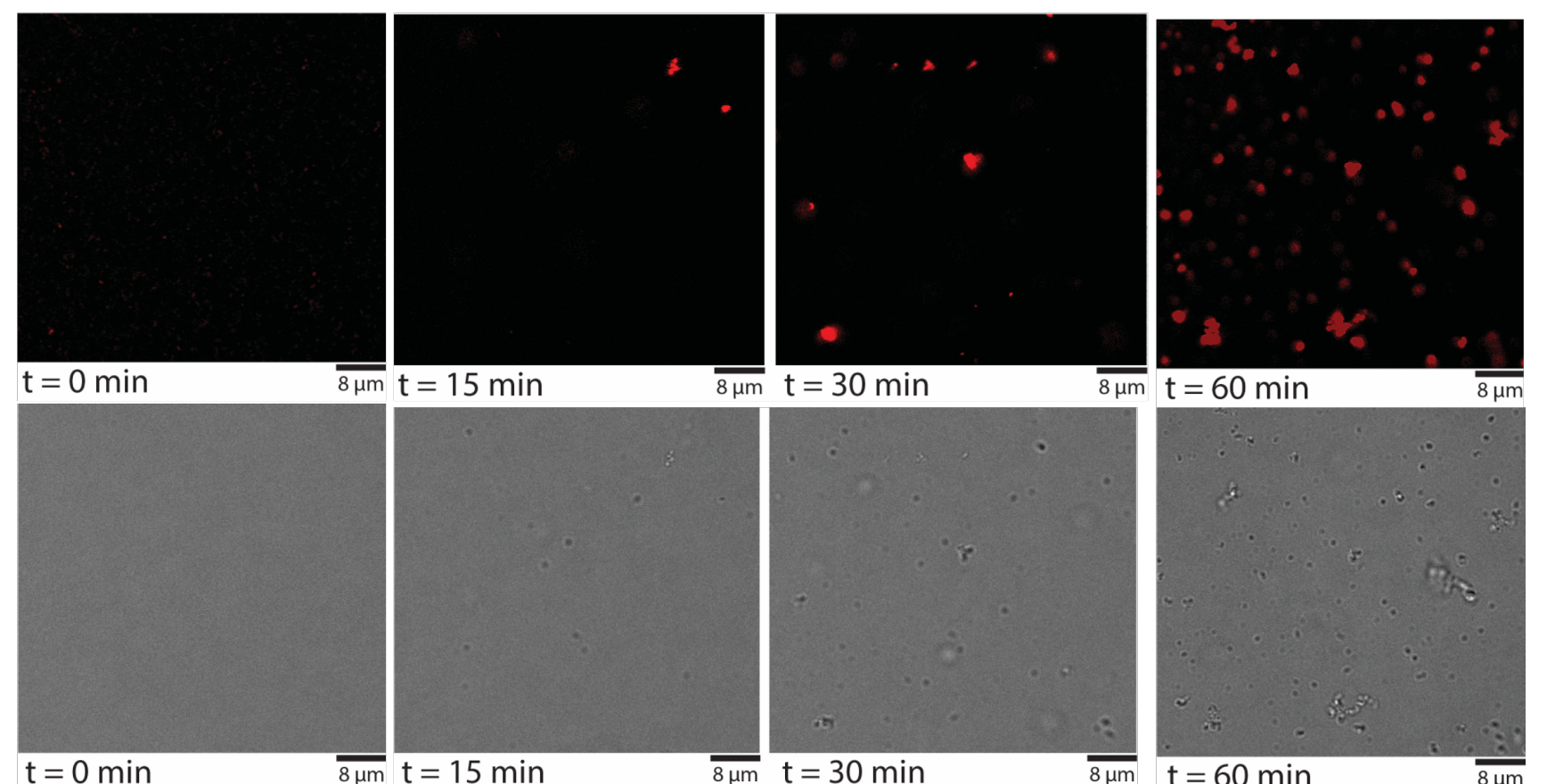

B

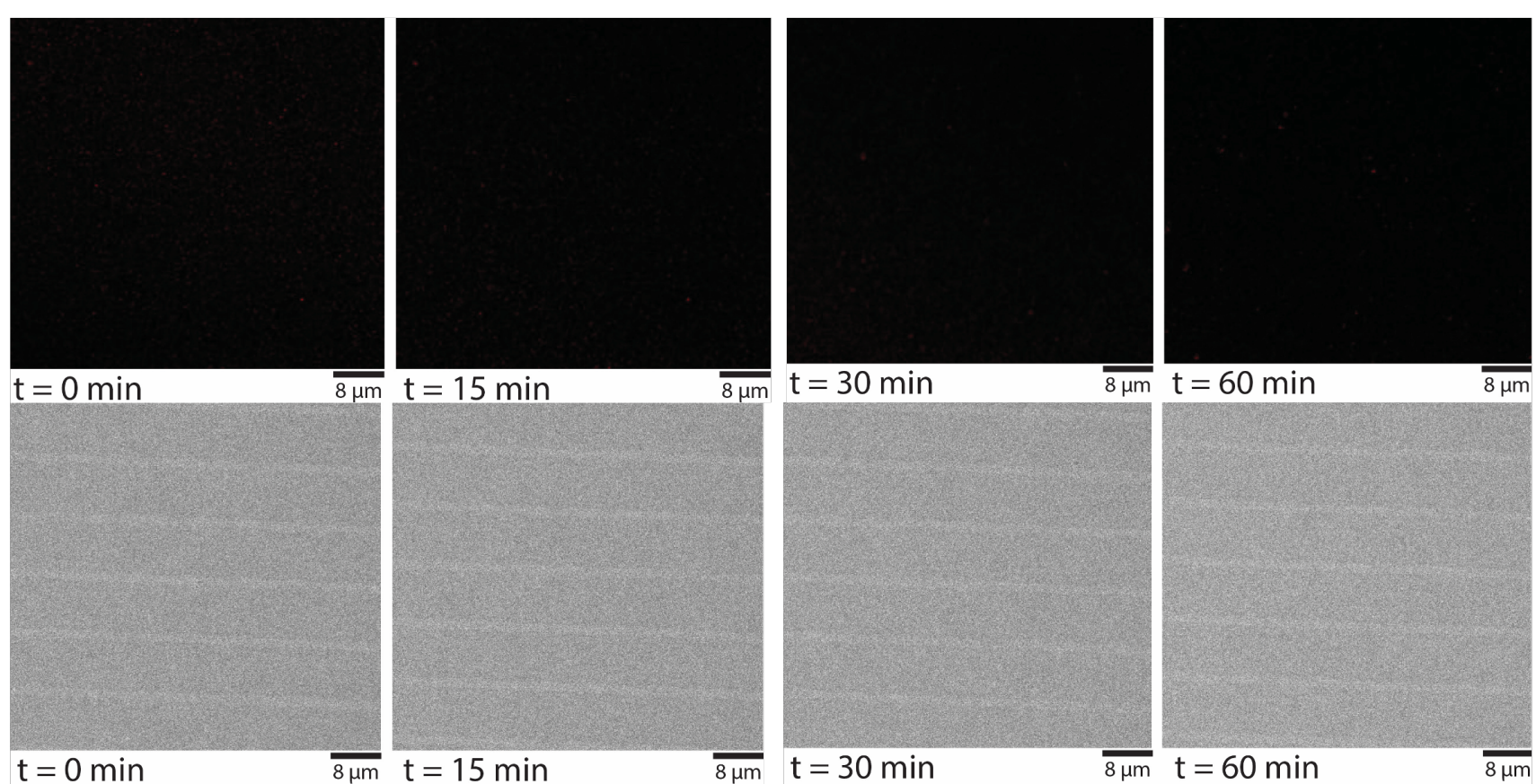

Figure S5. Confocal fluorescence microscopy images for 0-30\% 1a liposomes tagged with 0.08\% Rd-PE. A. Fluorescence and bright-field images for 30\% 1a liposomes at T= 0, 15, 30 and 60 minutes. Minute fluorescent dots were seen before zinc addition, while larger aggregates/fluorescent structures were seen after zinc addition. B. Fluorescence and bright-field images for $0 \% 1$ a liposomes at $T=0,15,30$ and 60 minutes. No significant changes in vesicle size and intensity were observed after zinc addition. 
A

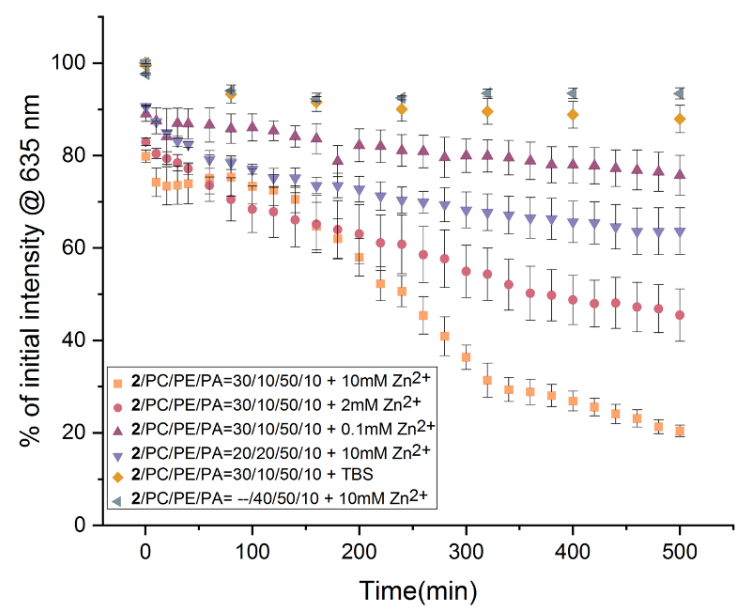

B

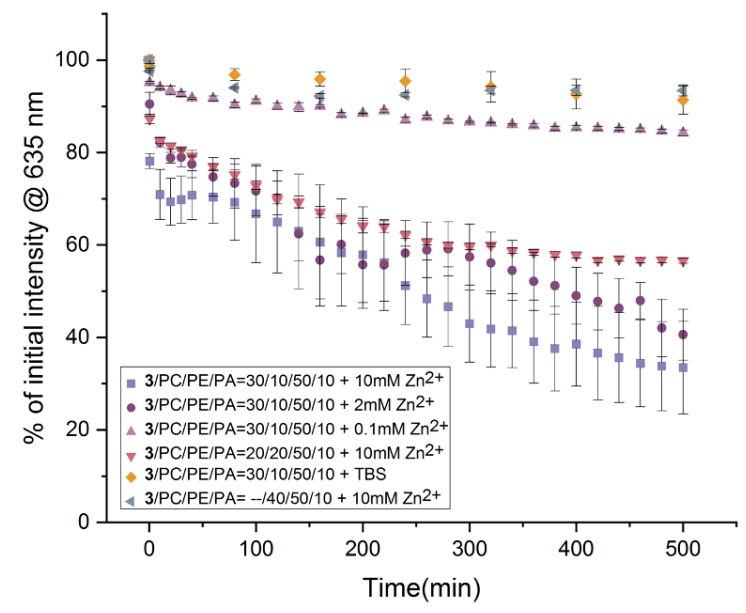

Figure S6. Time-dependent NR release profiles over time for ZRLs 2 (A) and $\mathbf{3}$ (B) at 0-30\% of liposome composition containing 50\% DOPE, 10\% PA, and PC as the remaining percentage. Error bars indicate standard error over triplicate experiments.

A

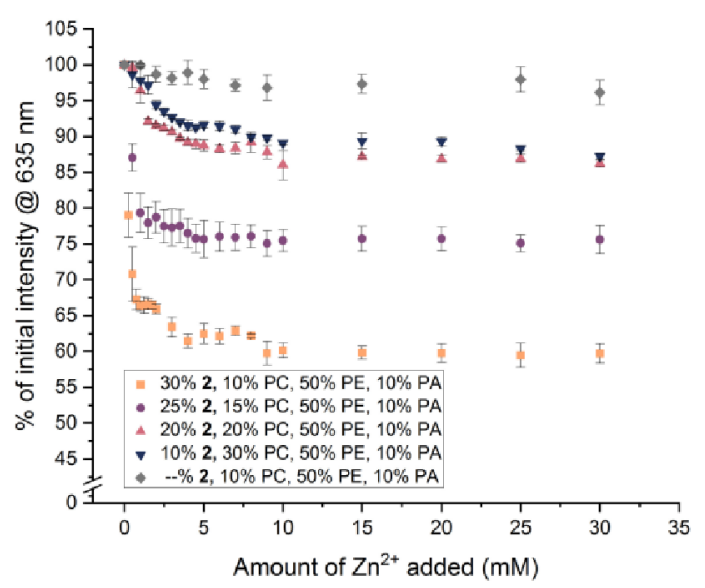

B

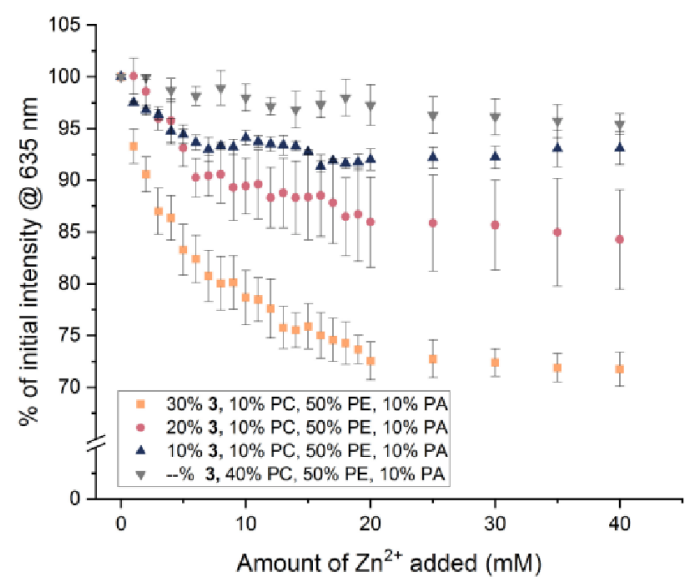

Figure S7. Results from NR titration experiments of liposomes containing ZRLs 2 (A) or $\mathbf{3}$ (B). Error bars indicate standard error over triplicate experiments. 
A

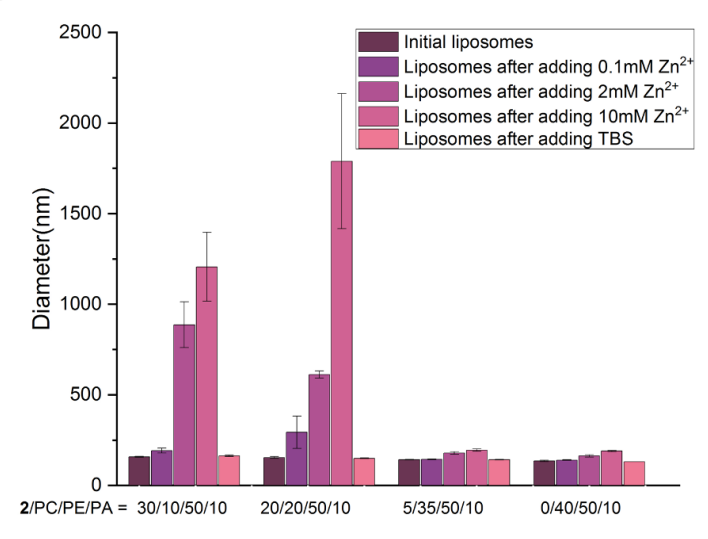

B

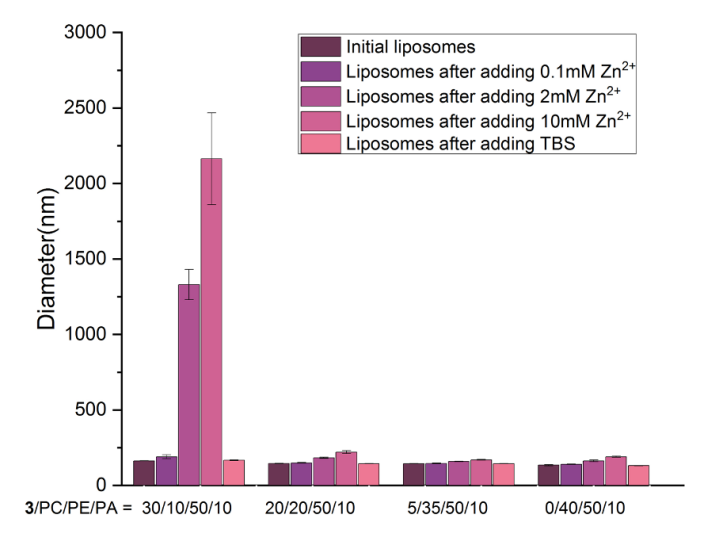

Figure S8. DLS analysis of liposomes containing $0-30 \%$ of $\mathbf{2}$ (A) or $\mathbf{3}$ (B) upon addition of varied zinc concentrations. A dose-dependent increase in size is observed upon addition of zinc to liposomes. Error bars indicate standard error over triplicate experiments.

A

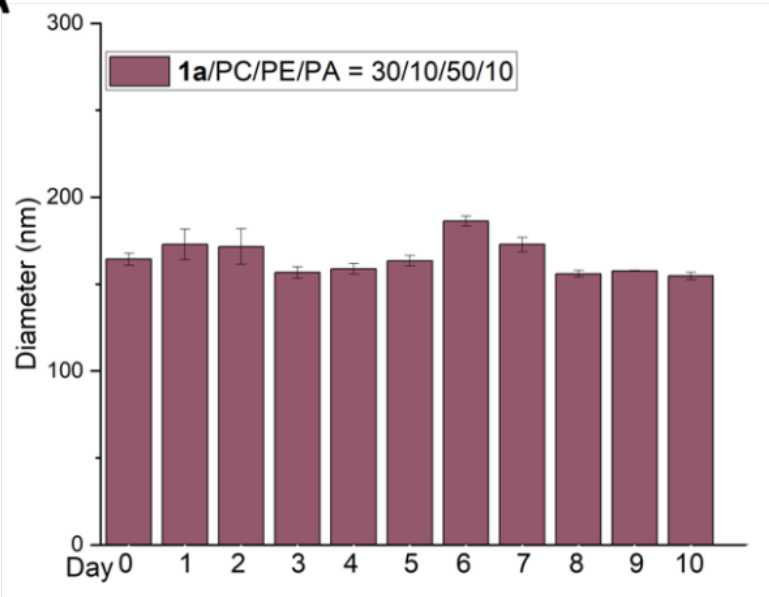

C

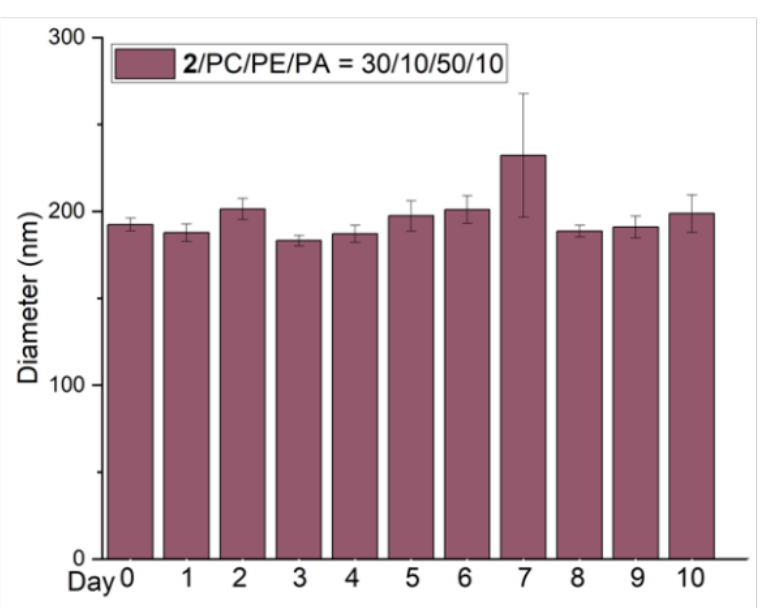

B

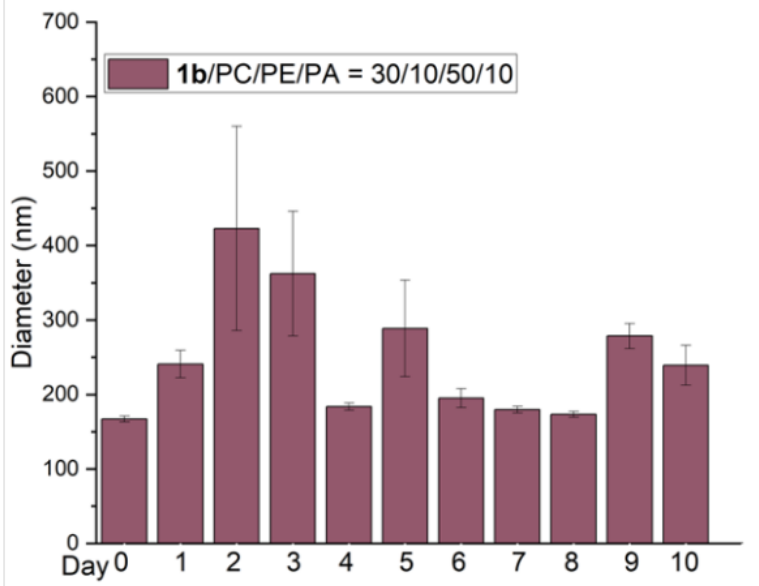

D

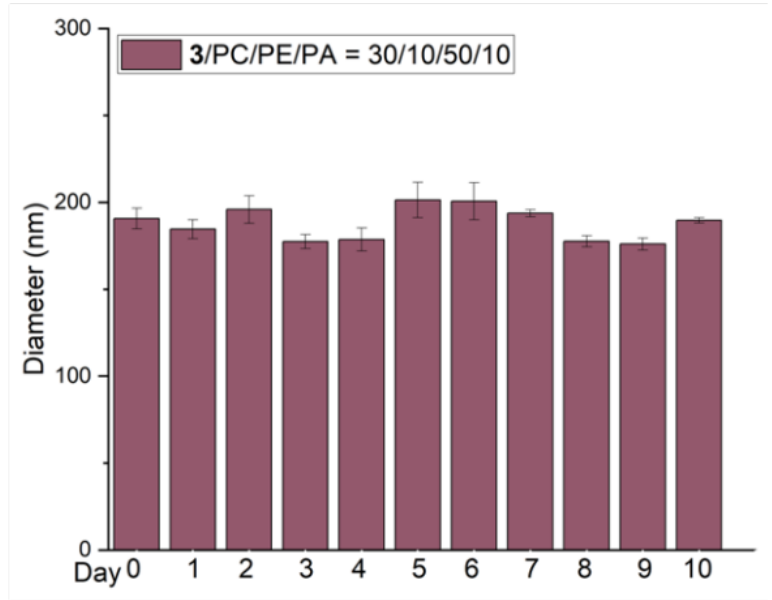

Figure S9. Liposome stability test for liposomes containing ZRL 1a (A), 1b (B), 2 (C), 3 (D). Liposomes containing 1a, $\mathbf{2}$ and $\mathbf{3}$ were found to be stable over a period of days while liposomes containing $\mathbf{1 b}$ showed gradual degradation particularly after 48 hours. 
A

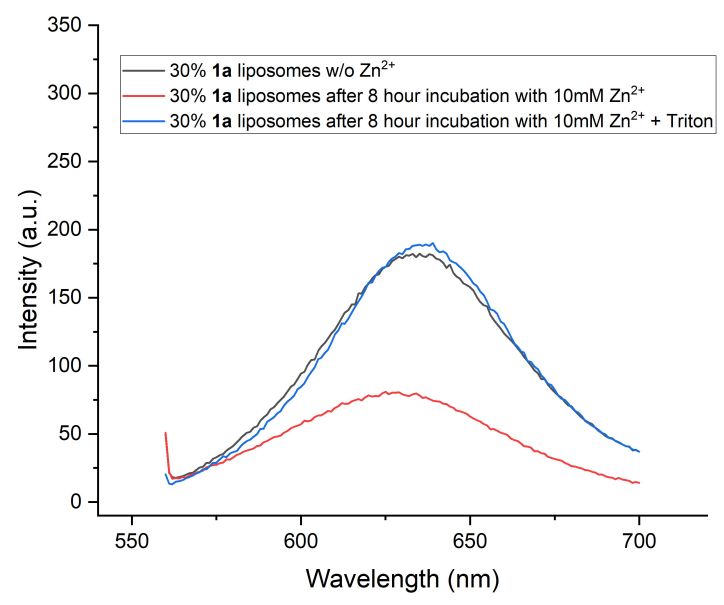

B

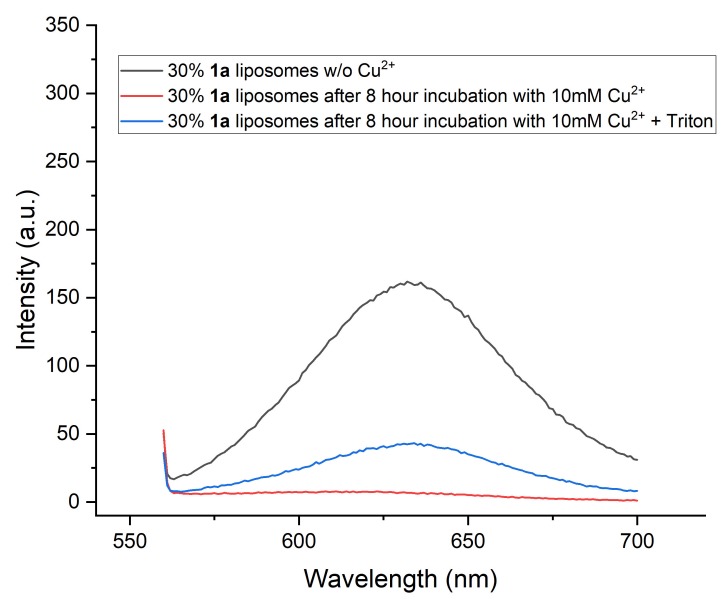

Figure S10. Triton X-100 restoration of NR fluorescence for 30\% 1a liposomes following treatment with $\mathrm{Zn}^{2+}(\mathbf{A})$ or $\mathrm{Cu}^{2+}$ (B). Both metal ions induced a significant amount of fluorescence decrease upon addition to liposomes. Complete restoration of fluorescence was observed for $30 \% 1$ a liposomes that had been incubated with zinc upon Triton addition while copper led to only a minor amount of fluorescence restoration upon Triton addition. 


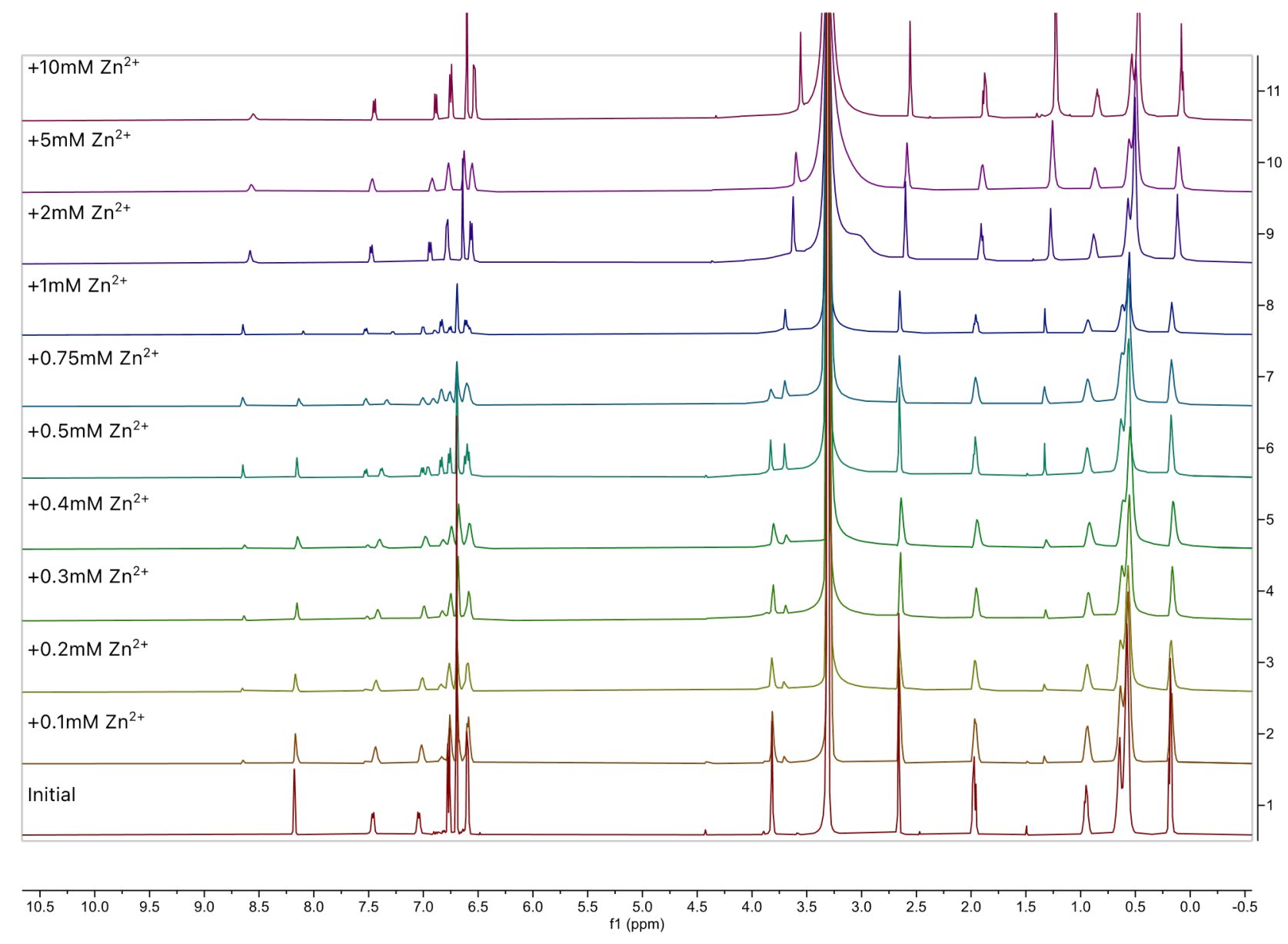

Figure S11. ${ }^{1} \mathrm{H}$ NMR titration experiment for lipid $1 \mathrm{a}$ in $20 \% \mathrm{CD}_{3} \mathrm{OD} / \mathrm{CDCl}_{3}$. Initial spectrum depicts lipid before zinc treatment. The subsequent traces showcase the NMR spectra after addition of corresponding amounts of zinc. 


\section{A Lipid 1a}

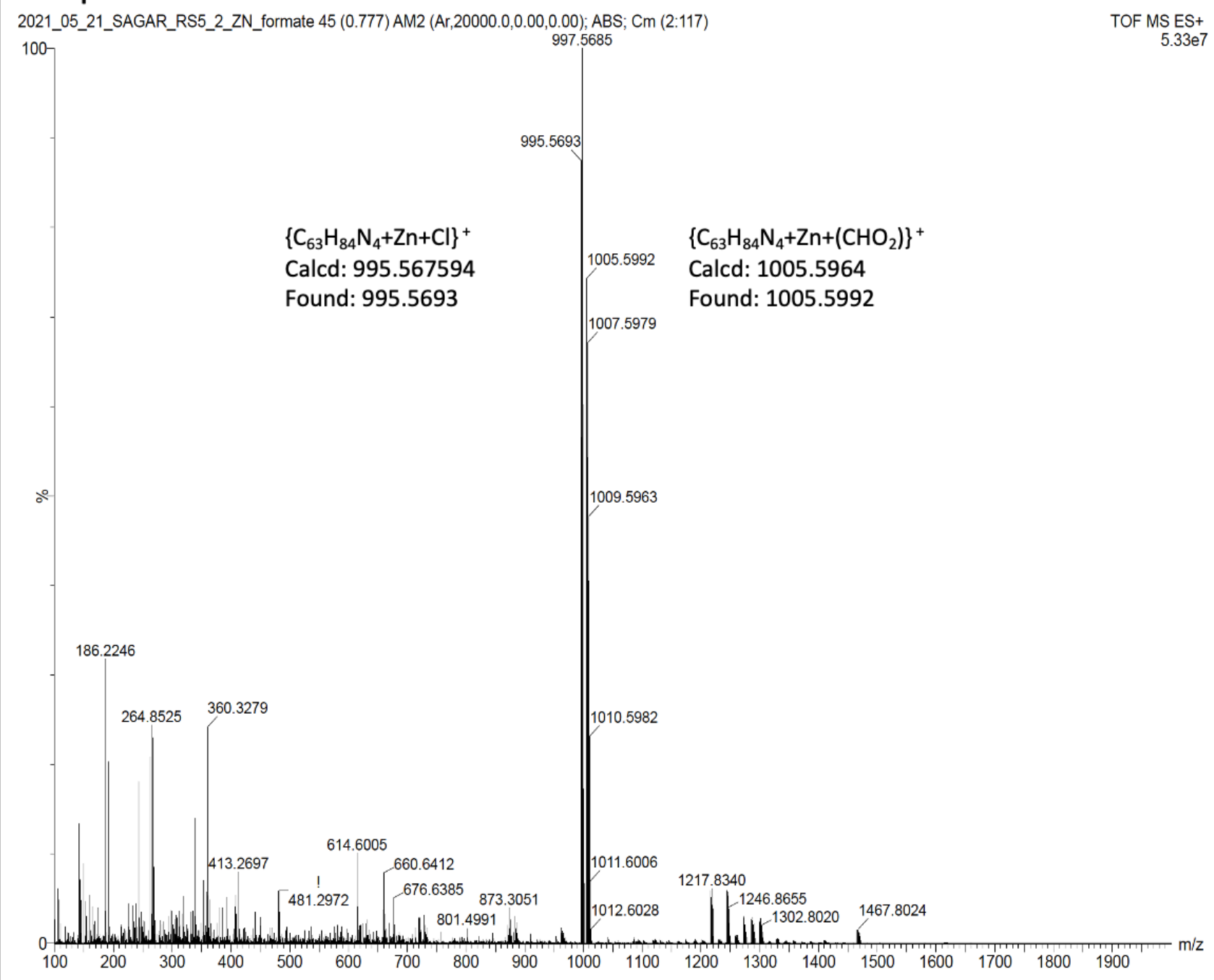




\section{B Lipid 1b}

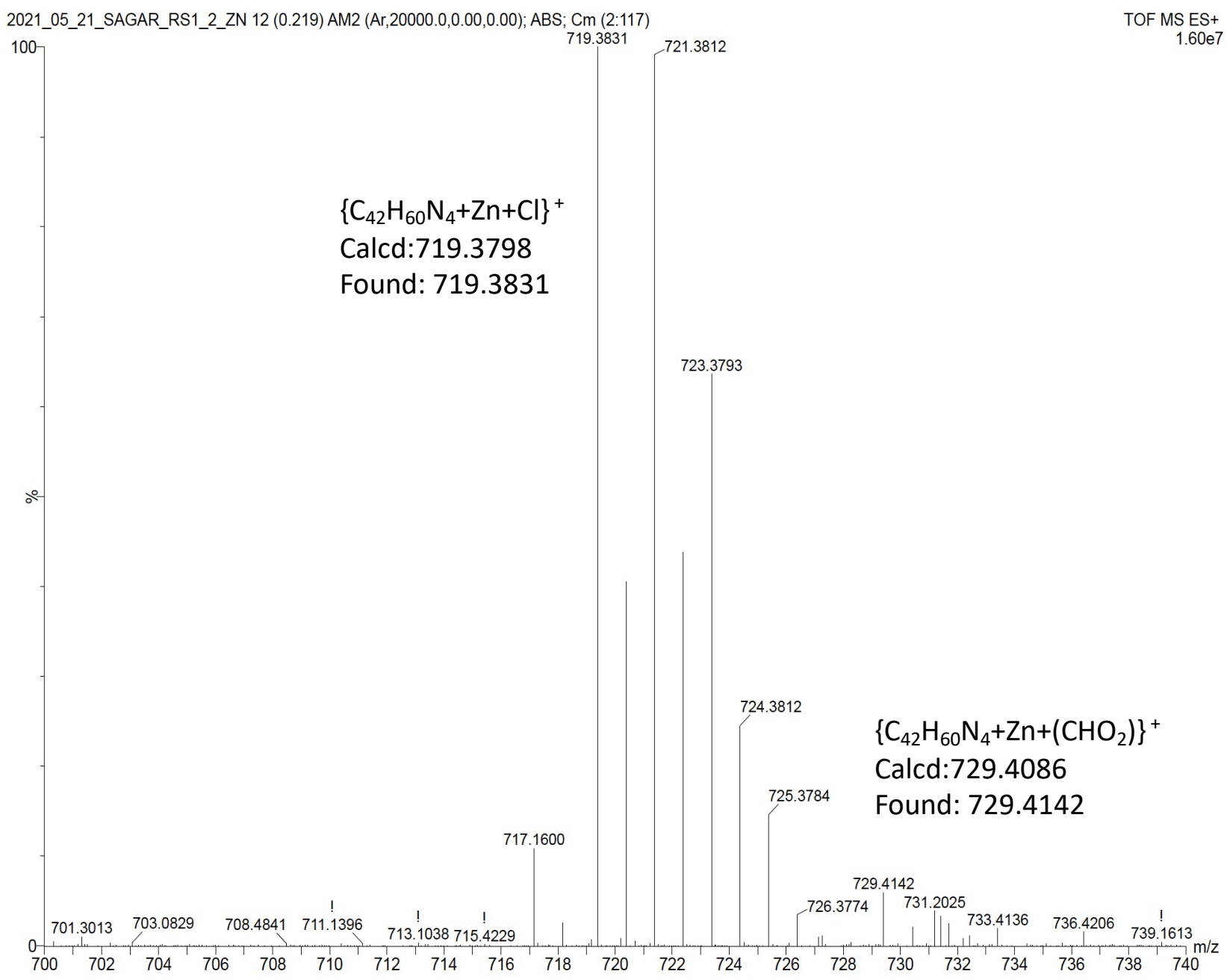




\section{Lipid 2}

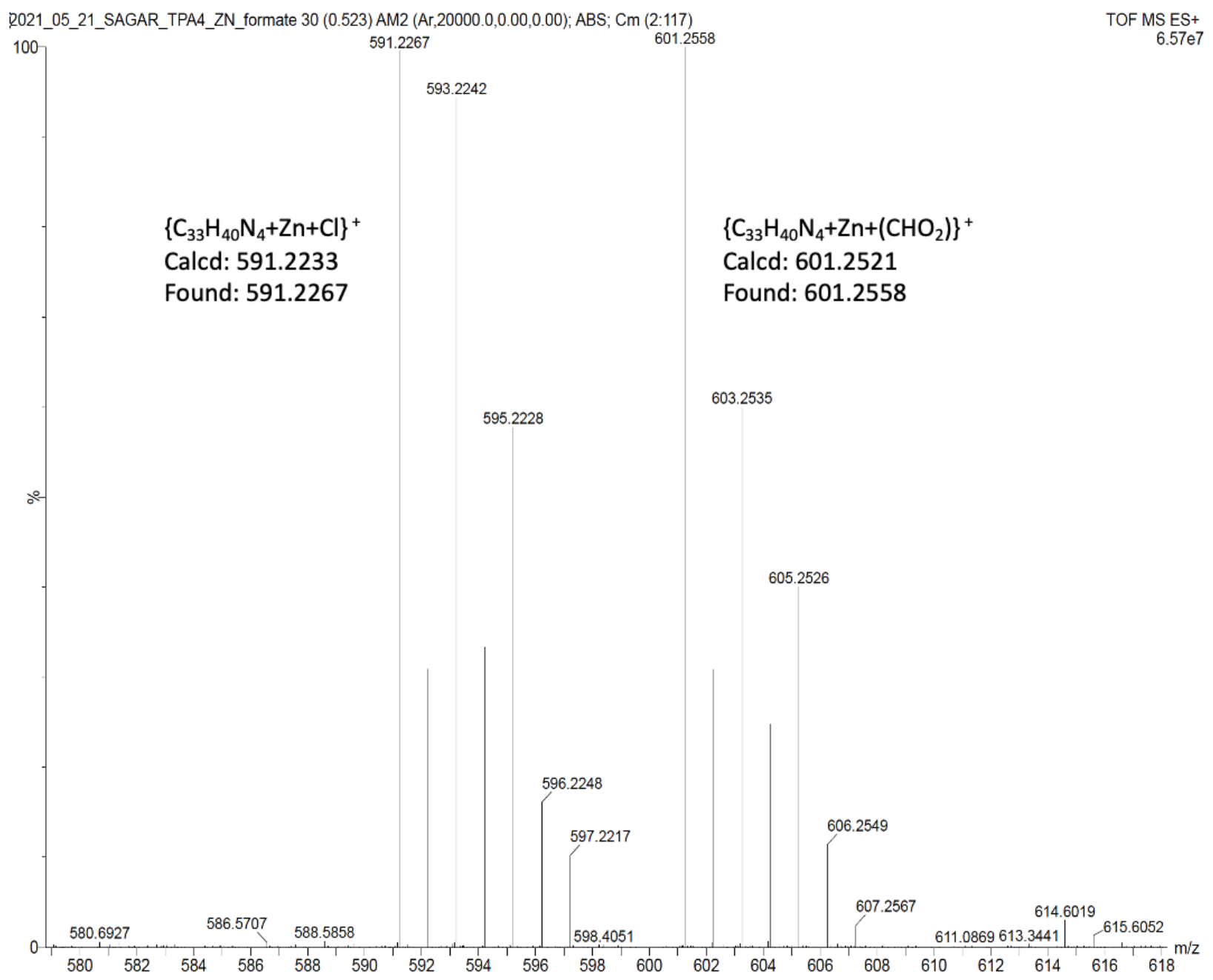




\section{Lipid 3}

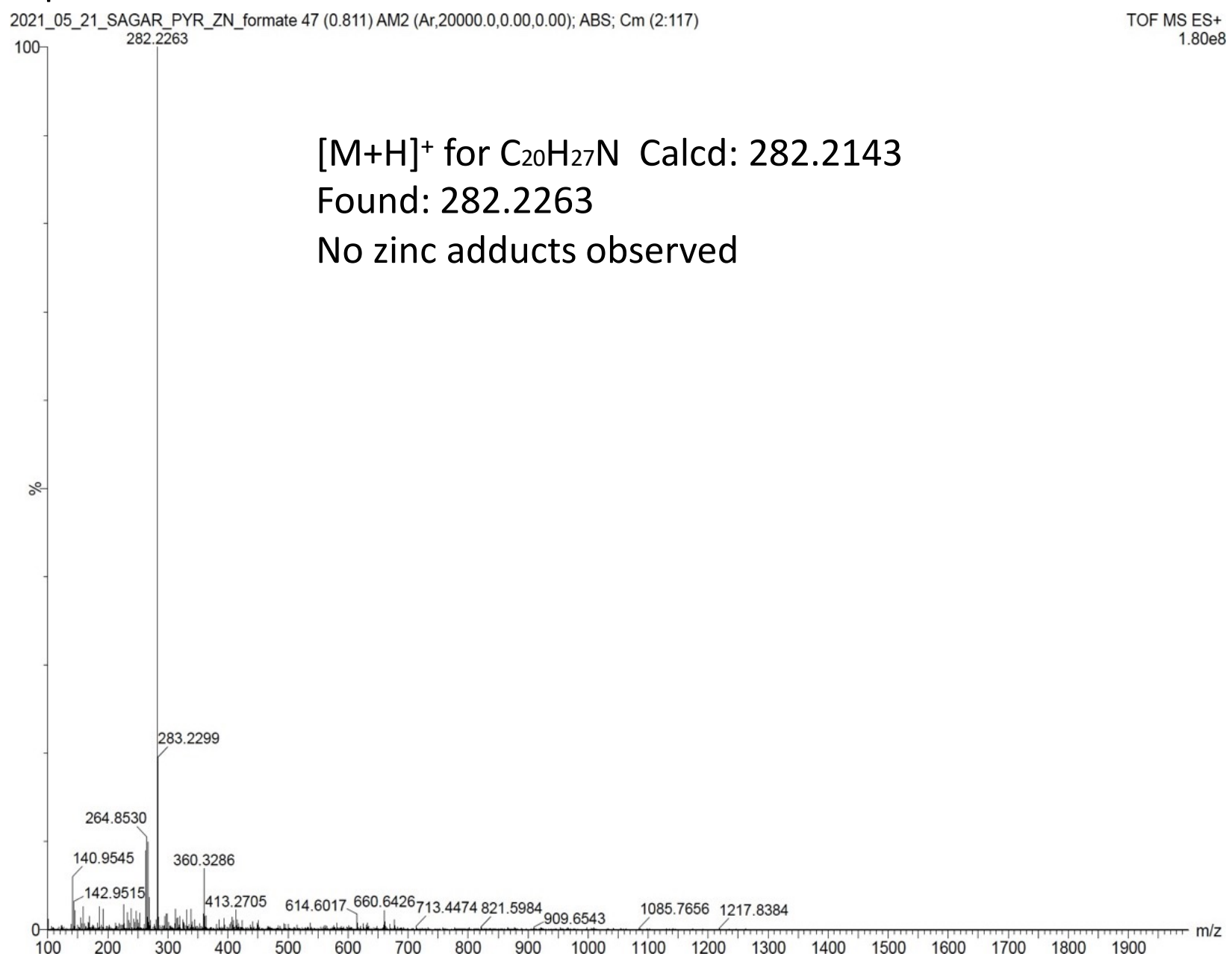

Figure S12. Mass spectra indicating formation of various zinc adducts with lipids $1 \mathbf{a}(\mathbf{A}), \mathbf{1 b}(\mathbf{B}), \mathbf{2}$ (C) and 3 (D). 


\begin{tabular}{|c|c|c|c|c|c|c|}
\hline \multicolumn{7}{|c|}{ Formulations explored for hydrophilic triggered release } \\
\hline Entry & Comments & $\begin{array}{c}\% \\
\text { ZRL } \\
2\end{array}$ & $\begin{array}{l}\% \\
\mathrm{PC}\end{array}$ & $\begin{array}{c}\% \\
\text { DOPE }\end{array}$ & $\begin{array}{c}\% \\
\text { PA }\end{array}$ & Outcome \\
\hline 1 & Ref. set & 30 & 10 & 50 & 10 & $\begin{array}{c}\text { Did not produce stable } \\
\text { liposomes }\end{array}$ \\
\hline 2 & Varying ZRL & 20 & 20 & 50 & 10 & $\begin{array}{l}\text { Stable liposomes } \\
\text { Minimal release }\end{array}$ \\
\hline 3 & \multirow{4}{*}{$\begin{array}{c}\text { Fix 30\% ZRL and } \\
\text { 10\% PA } \\
\text { Varying DOPE and } \\
\text { PC }\end{array}$} & 30 & 20 & 40 & 10 & $\begin{array}{c}\text { Did not produce stable } \\
\text { liposomes }\end{array}$ \\
\hline 4 & & 30 & 30 & 30 & 10 & $\begin{array}{c}\text { Did not produce stable } \\
\text { liposomes }\end{array}$ \\
\hline 5 & & 30 & 50 & 10 & 10 & $\begin{array}{c}\text { Did not produce stable } \\
\text { liposomes }\end{array}$ \\
\hline 6 & & 30 & 40 & 20 & 10 & $\sim 64 \%$ release over 12 hours. \\
\hline
\end{tabular}

Table S2. Summary of formulations explored for hydrophilic triggered release using ZRL 2 containing different percentages of PC, DOPE, and PA. 


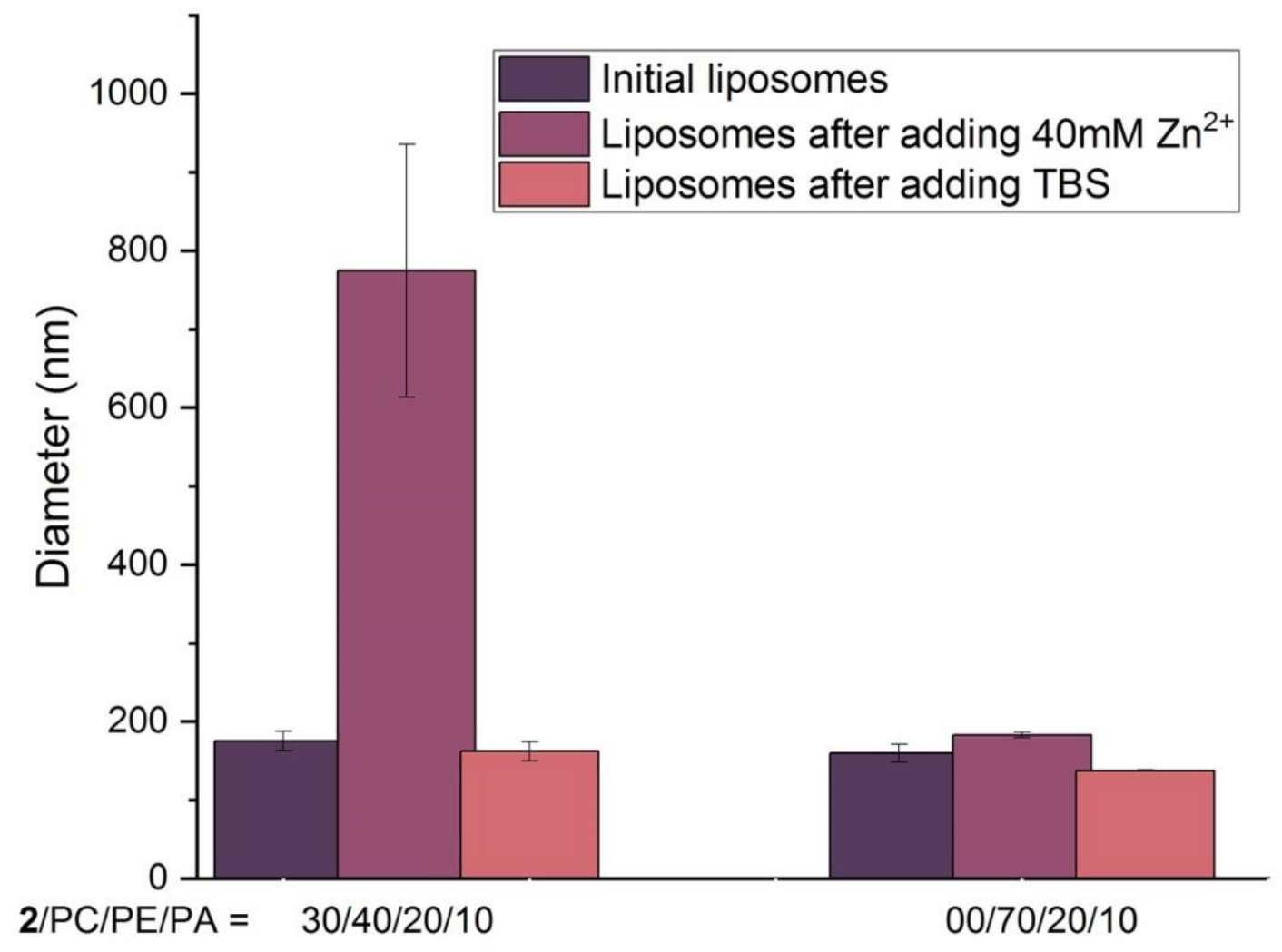

Figure S13. DLS analysis of liposomes containing 0-30\% of $\mathbf{2}$ upon addition of varied zinc concentrations. Error bars indicate standard error over triplicate experiments. 


\section{${ }^{1} \mathrm{H}$ NMR}

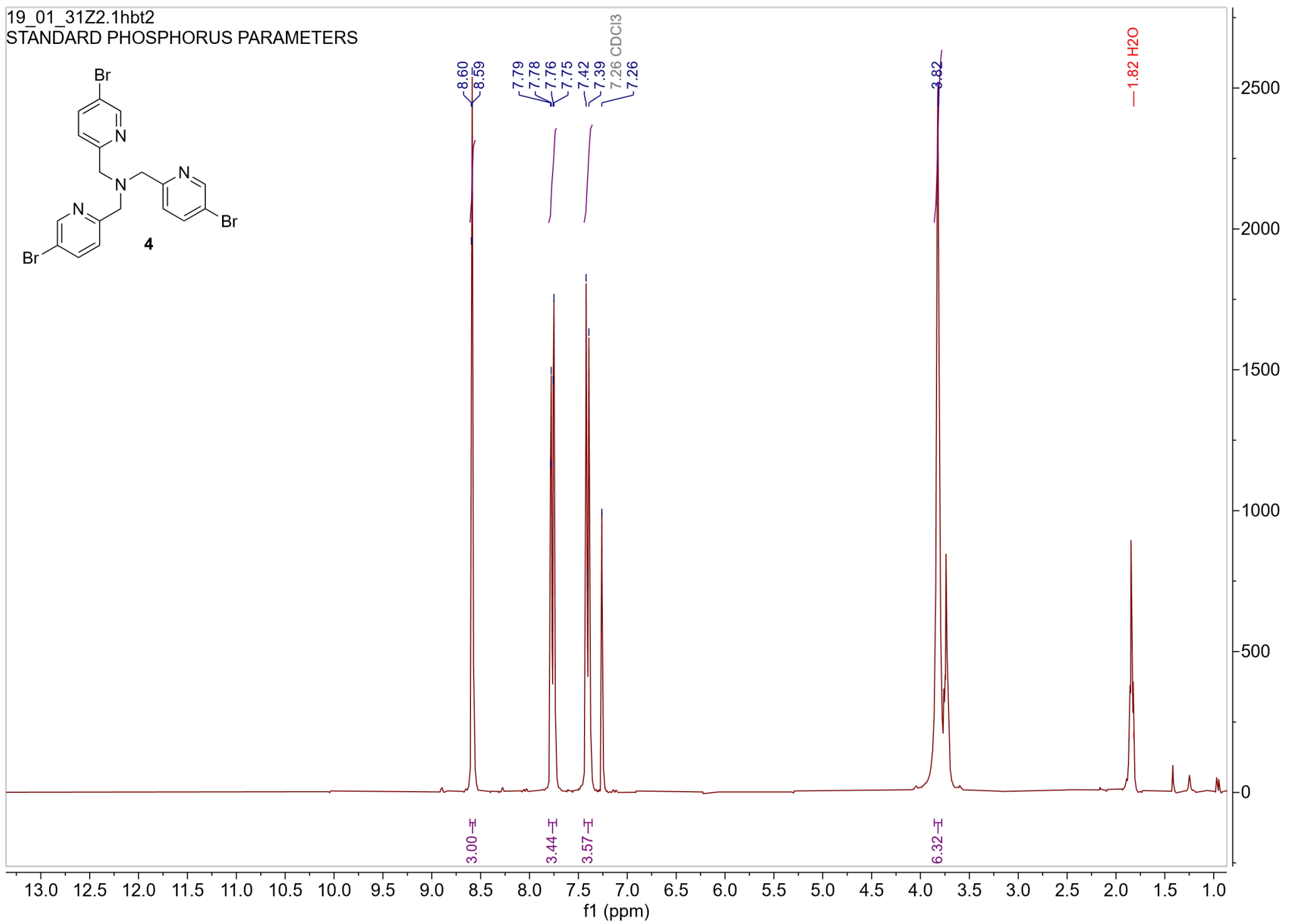




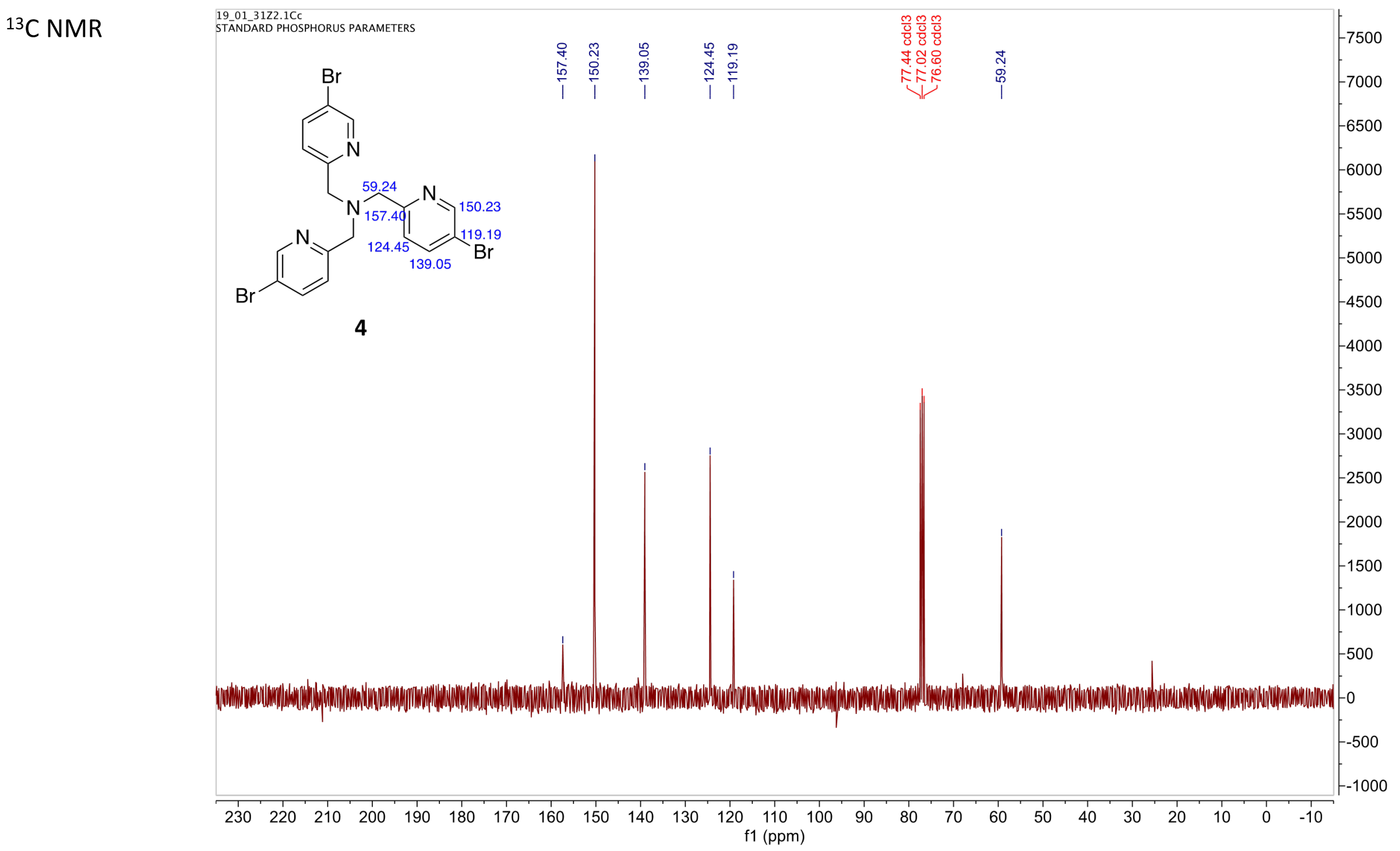


${ }^{1} \mathrm{H} N M R$

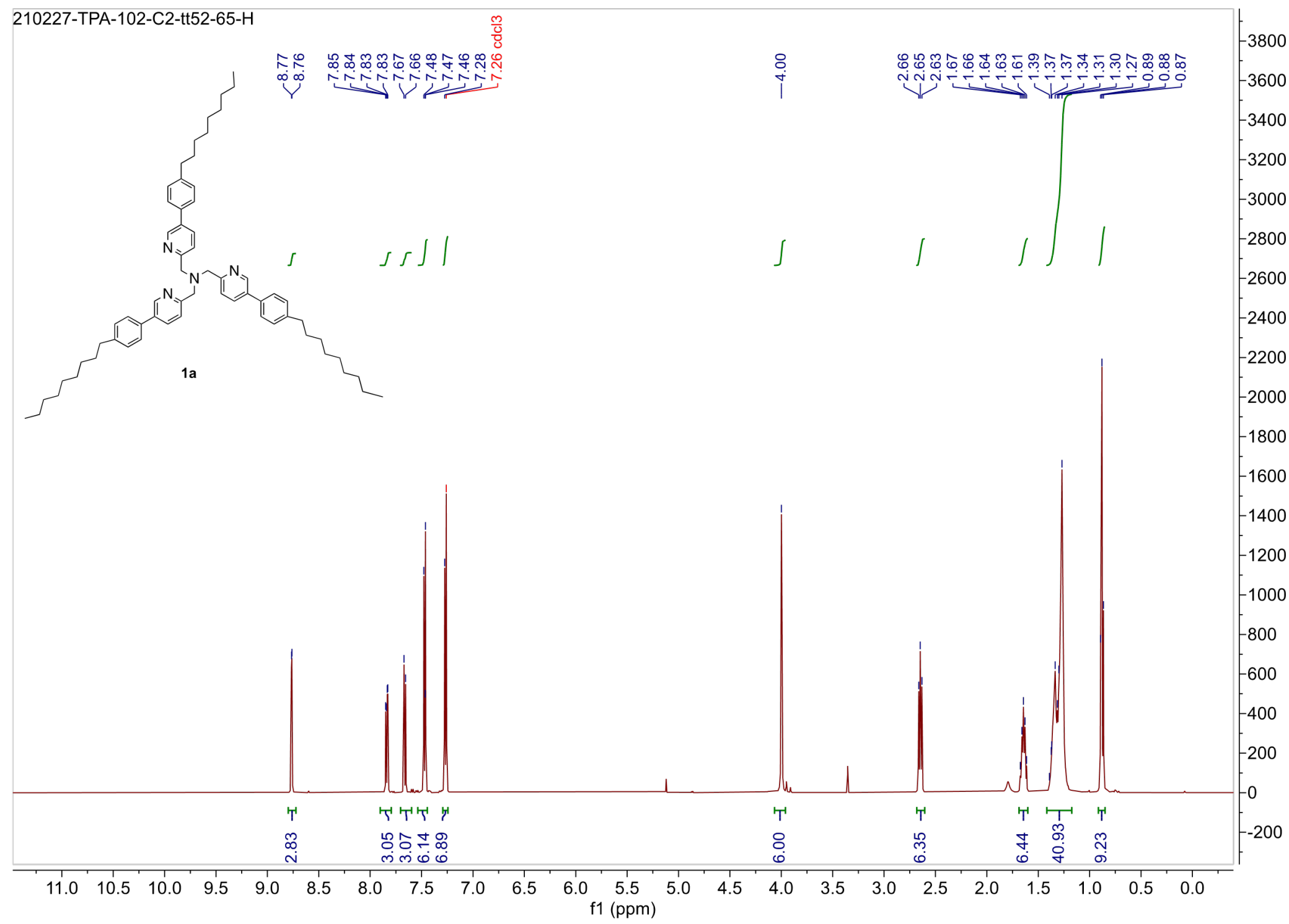




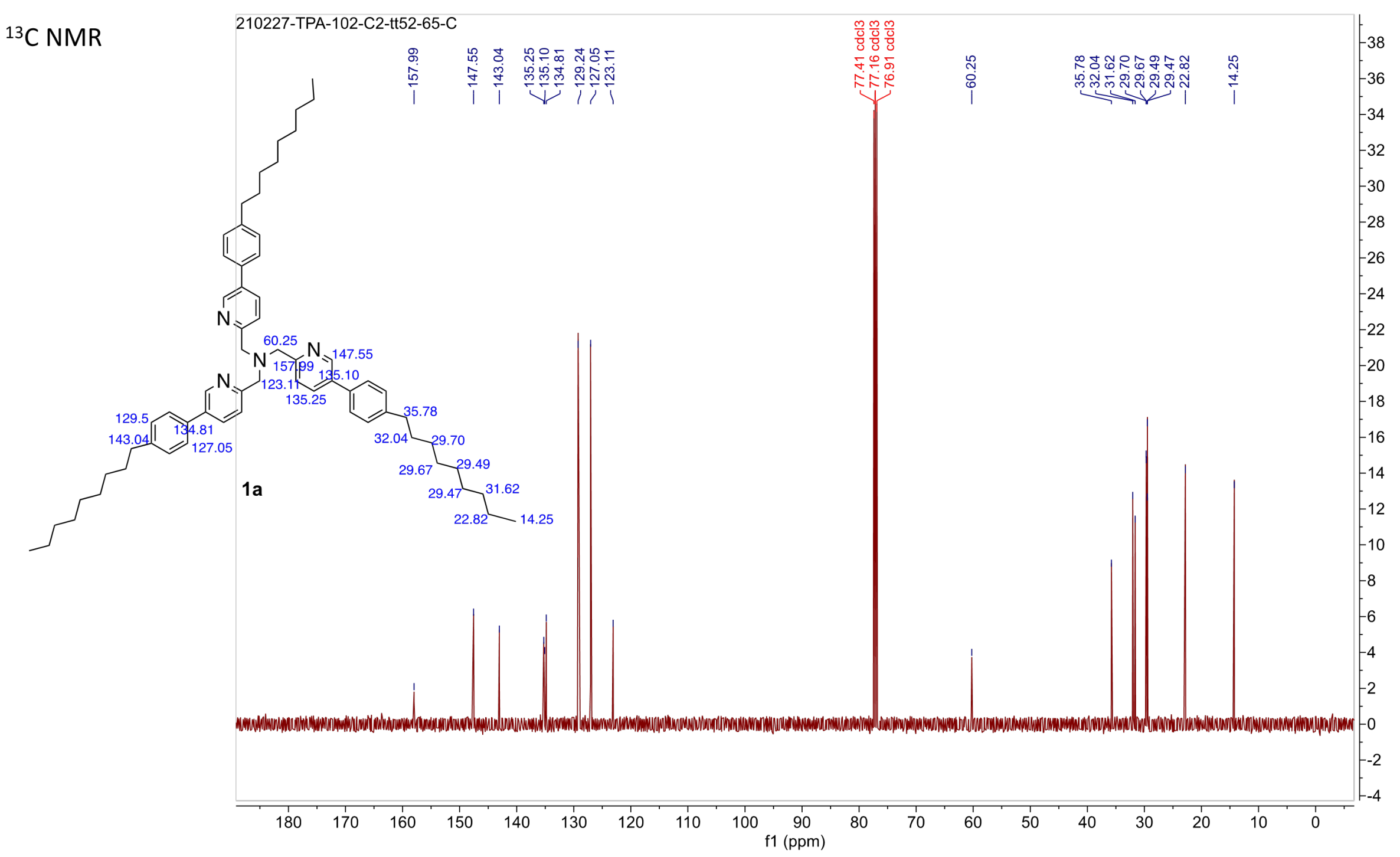




\section{ESI-MS}

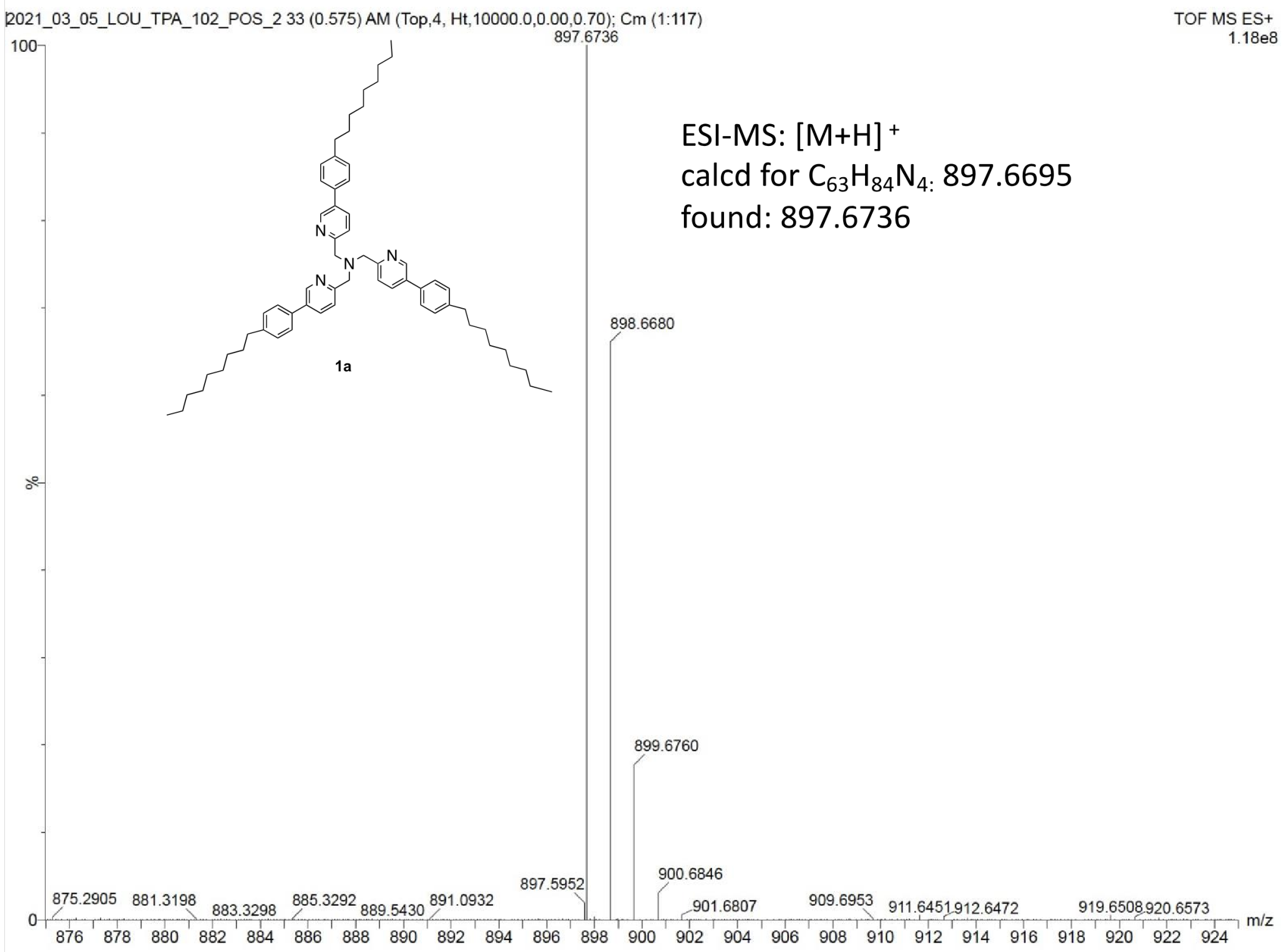




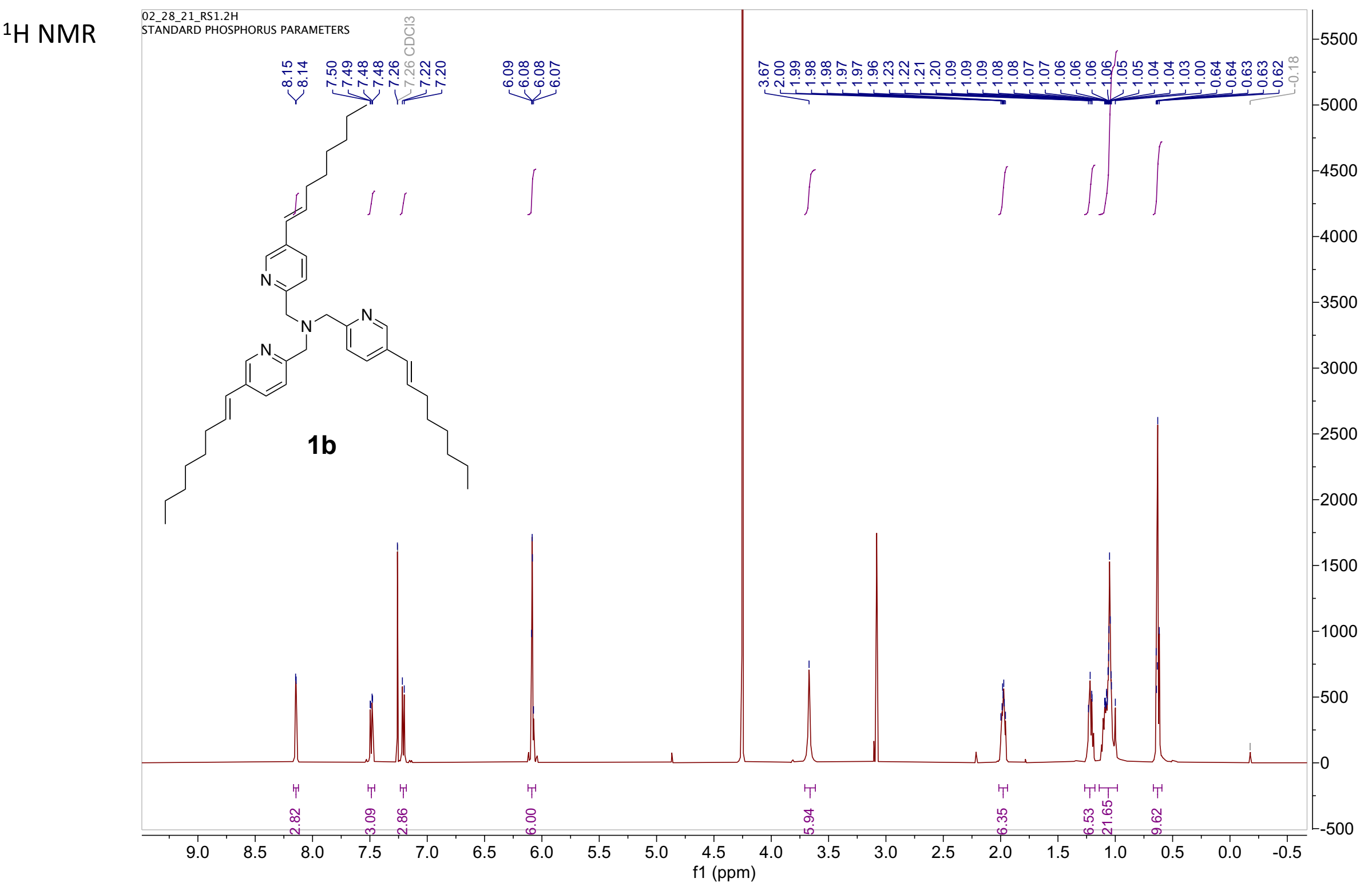




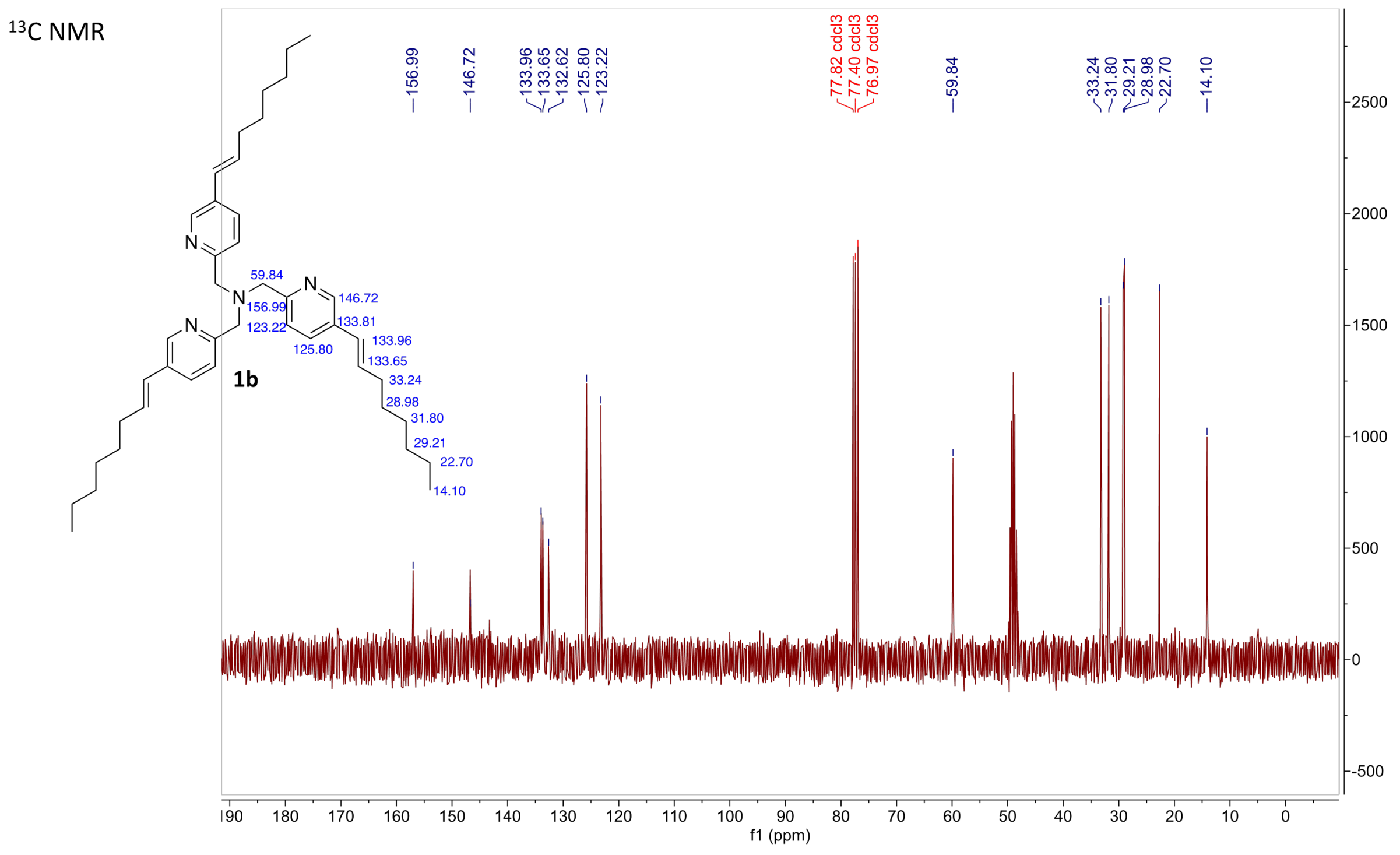




\section{ESI-MS}

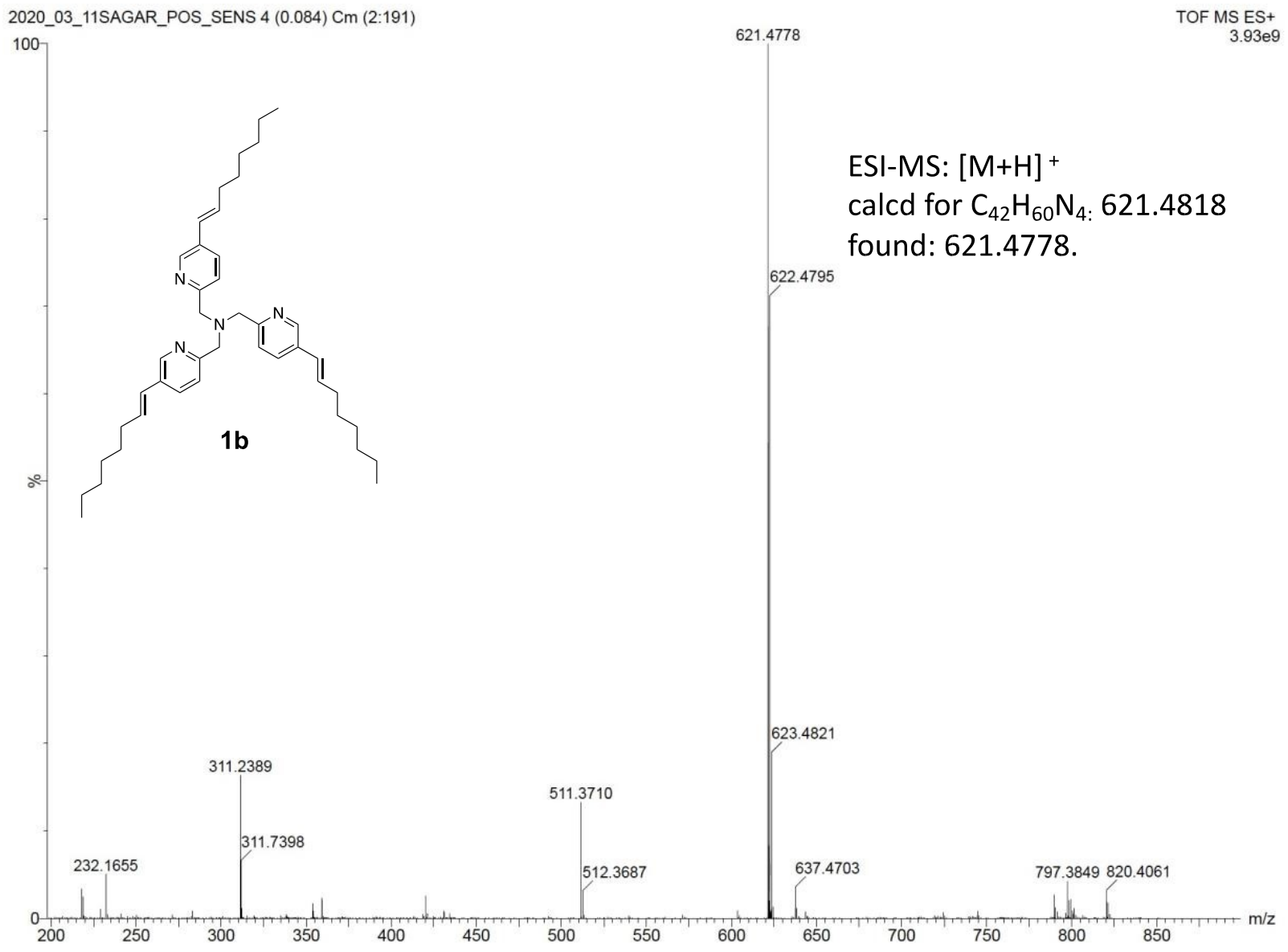


${ }^{1} \mathrm{H}$ NMR

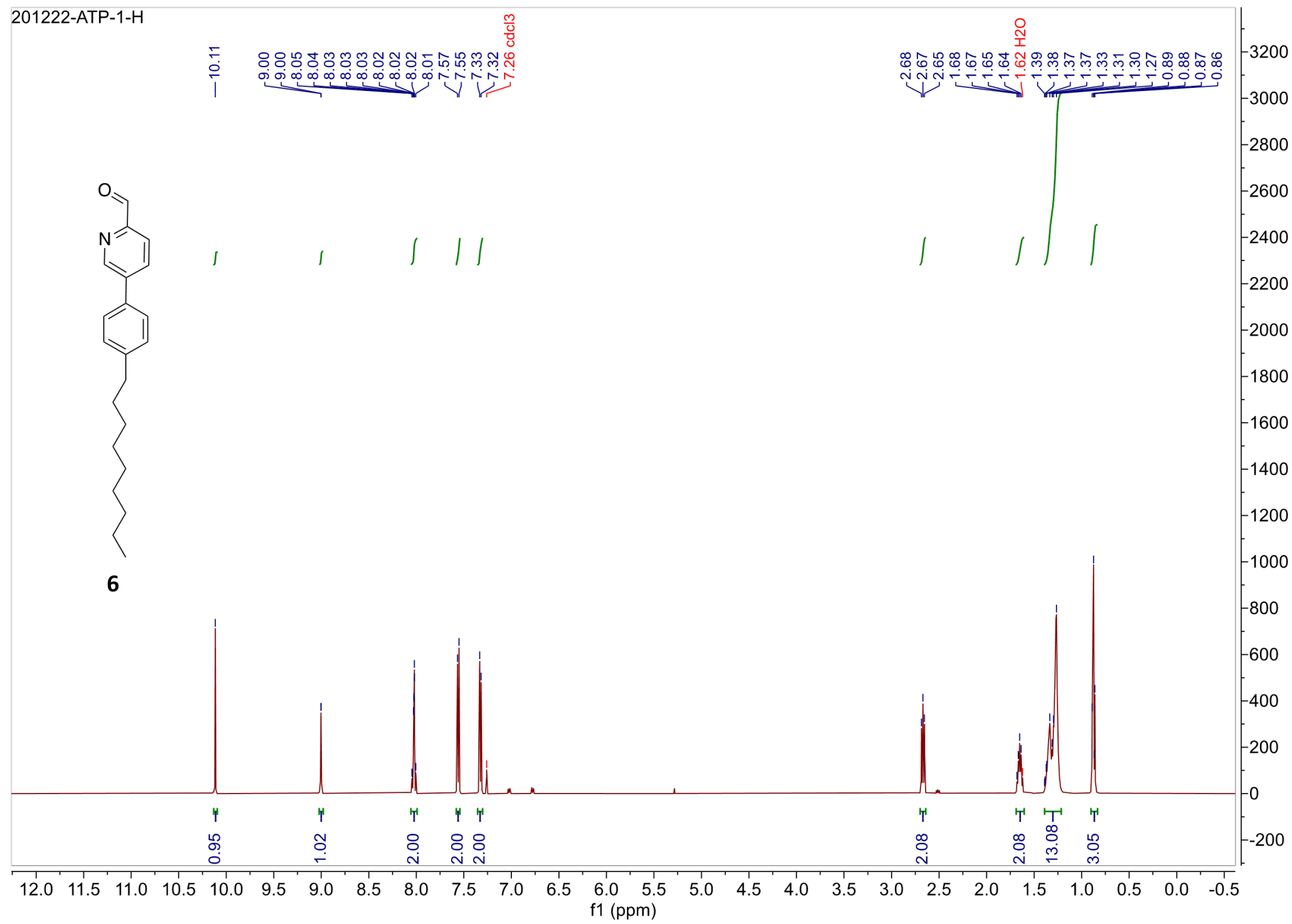




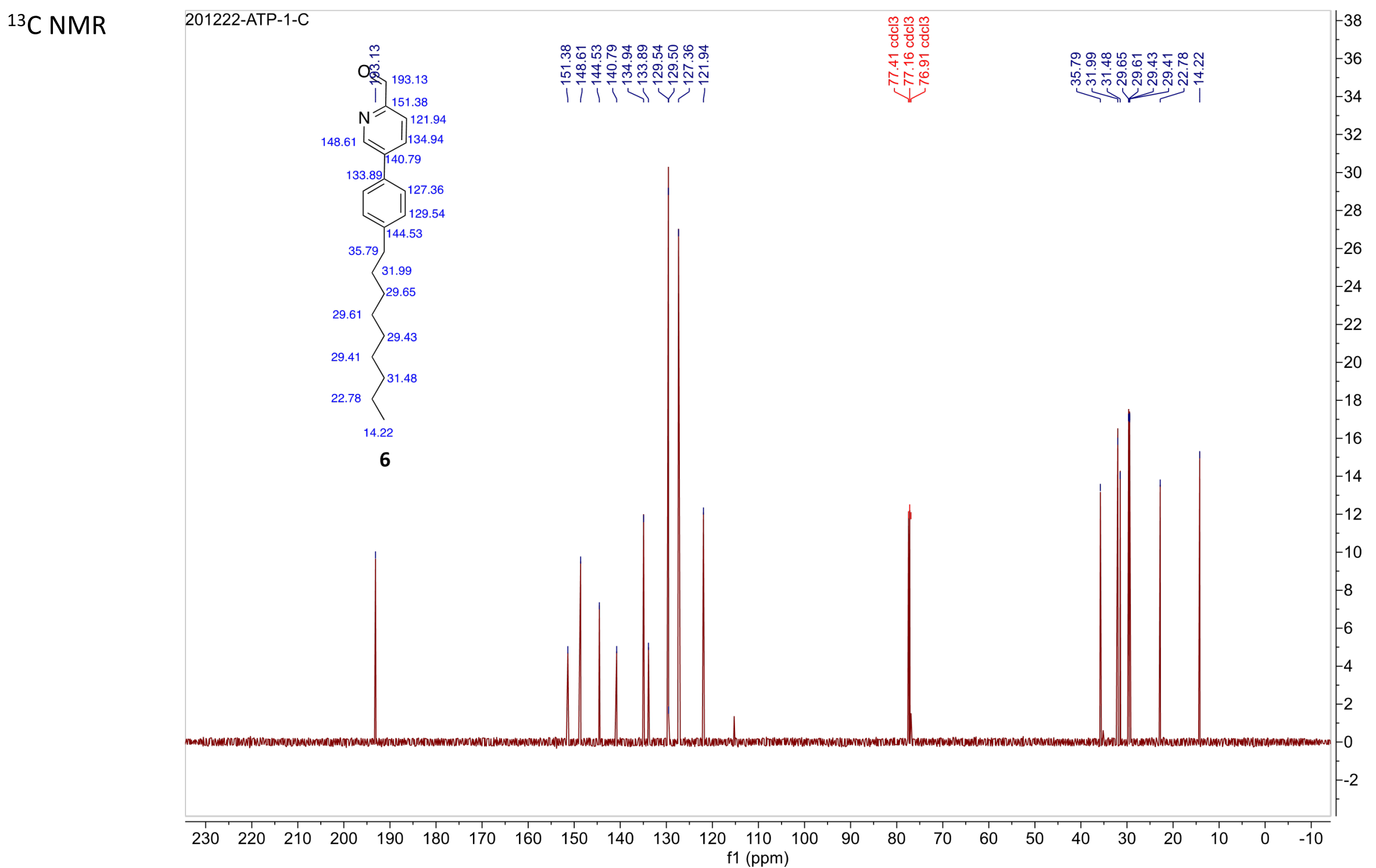


${ }^{1} \mathrm{H} N M R$

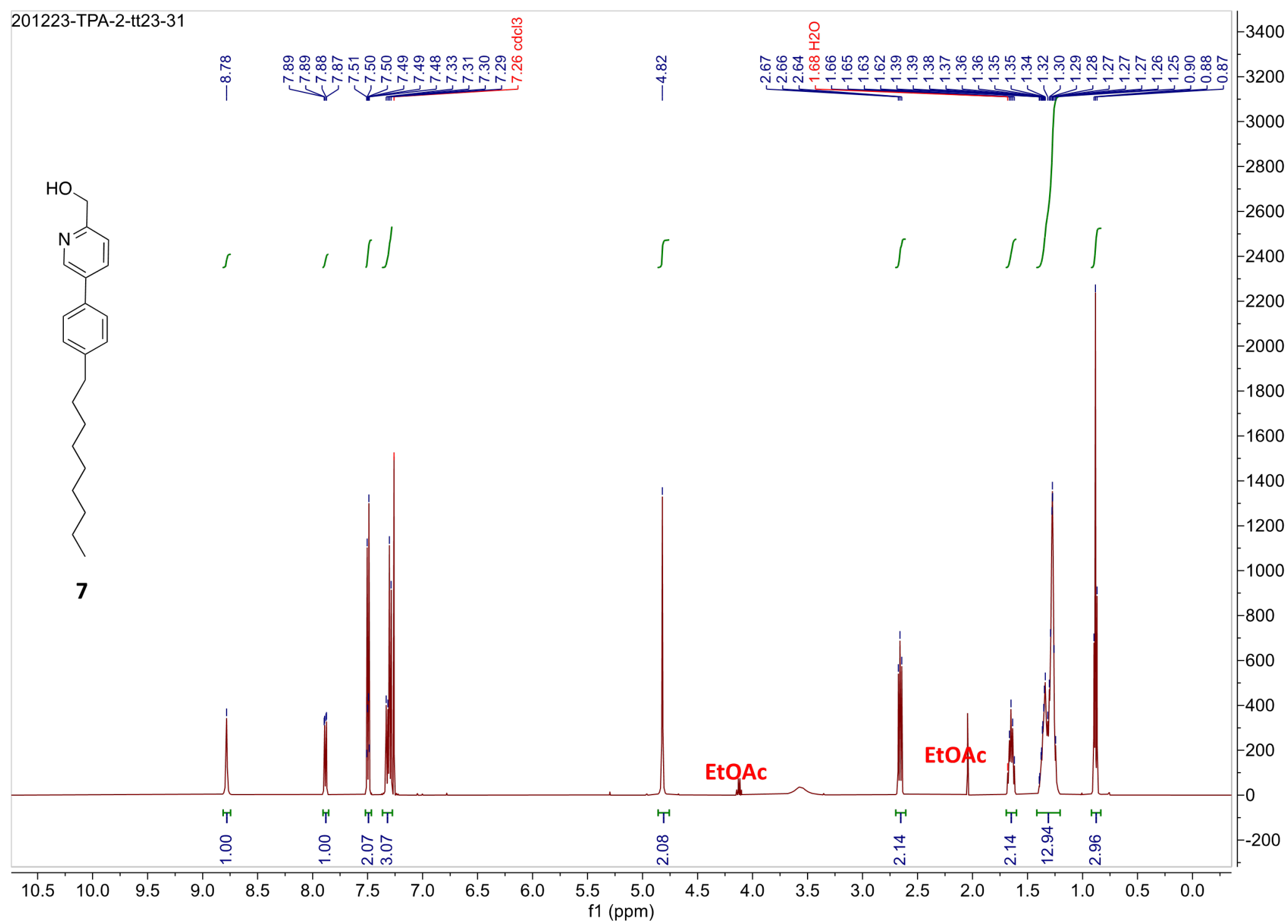


${ }^{13} \mathrm{C} N M R$

201223-TPA-2-tt23-31-C

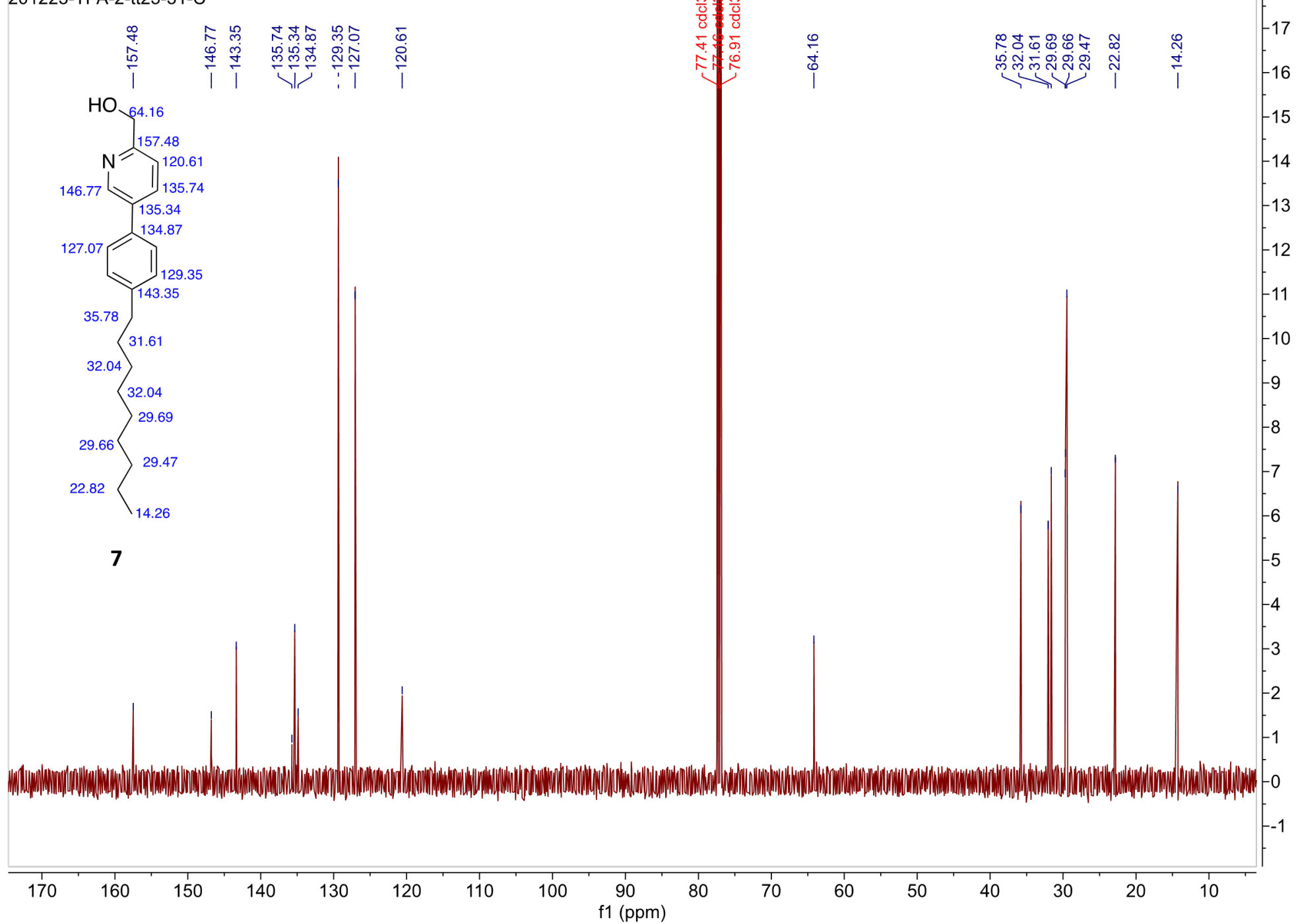




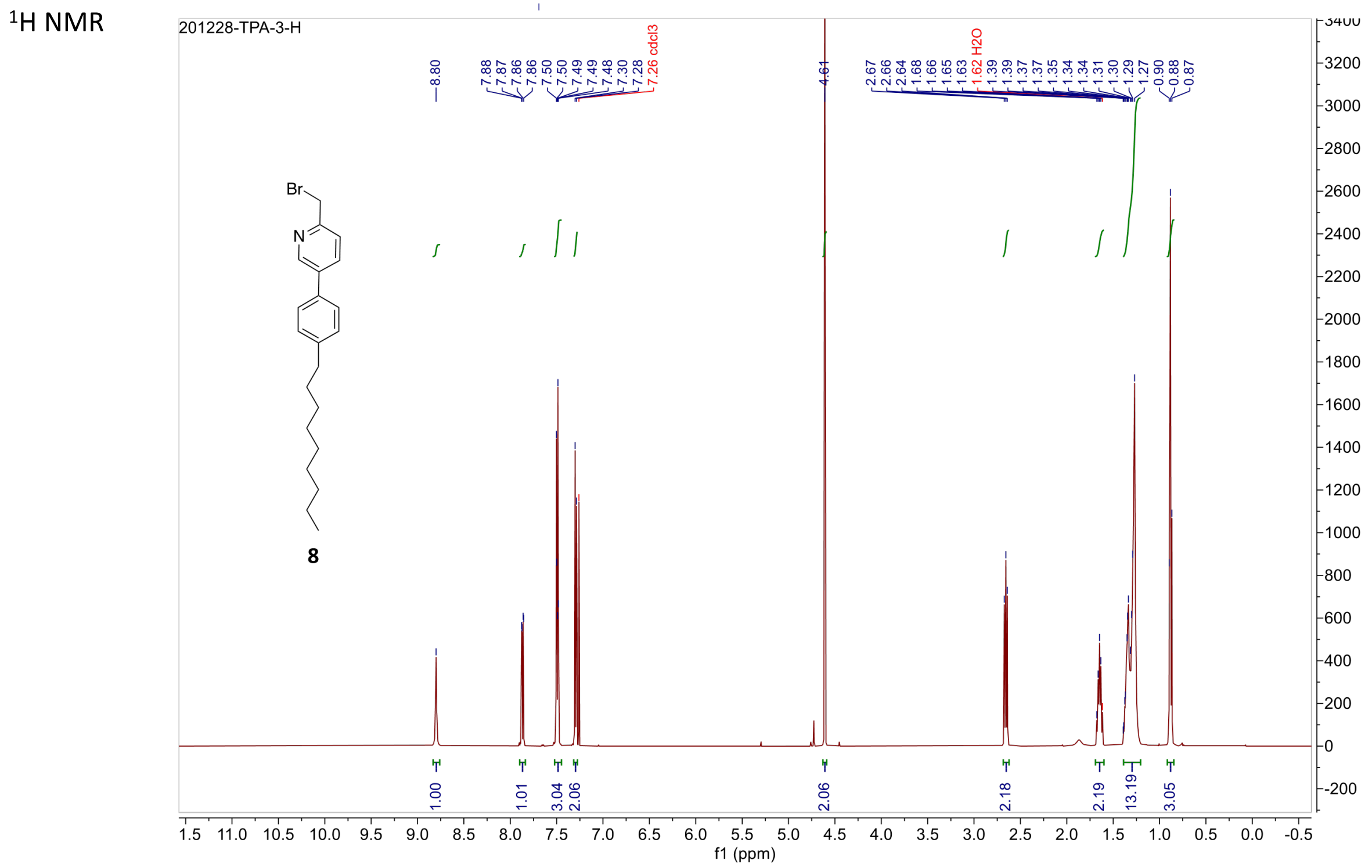


${ }^{13}$ C NMR

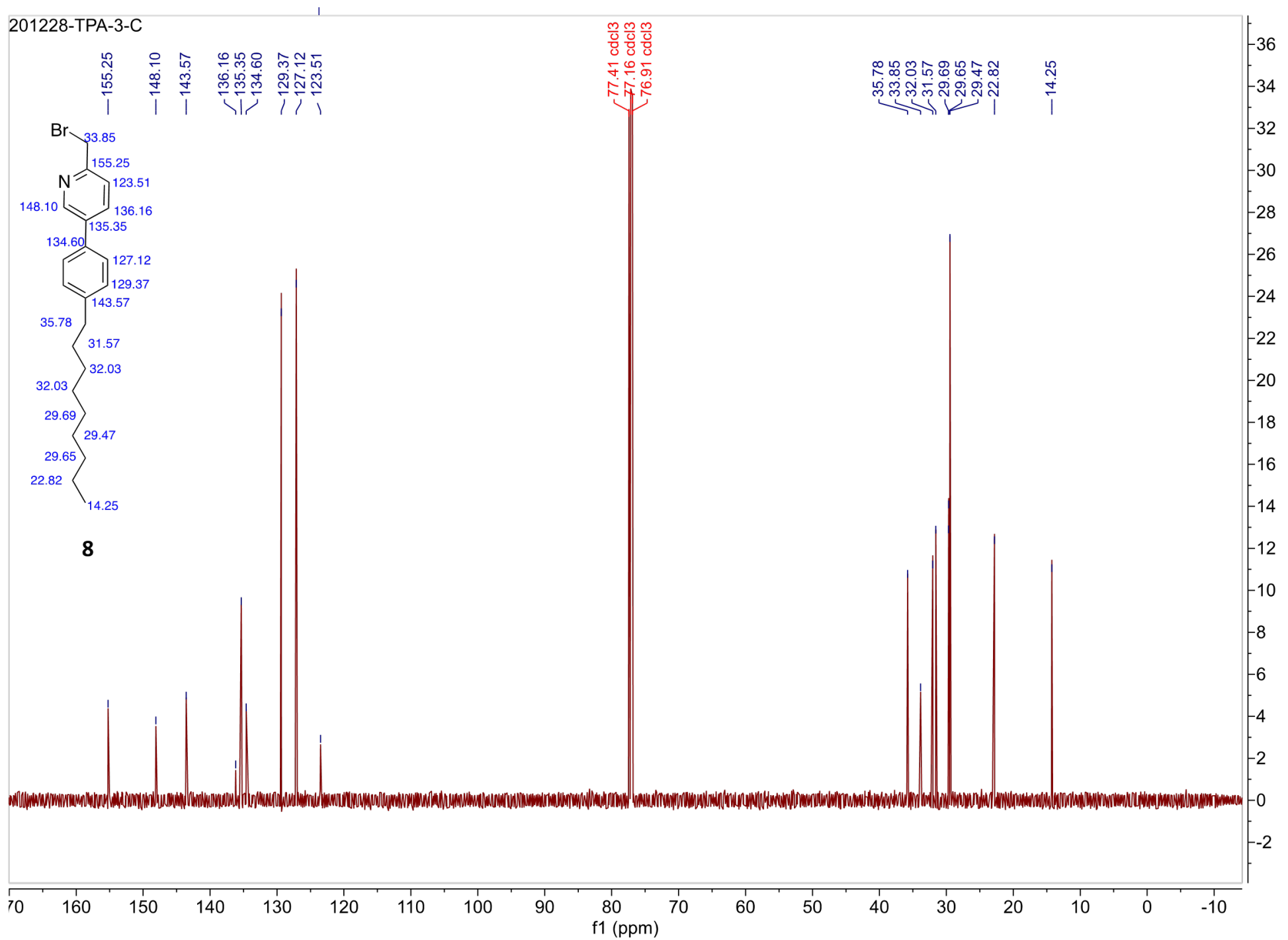


${ }^{1} \mathrm{H}$ NMR

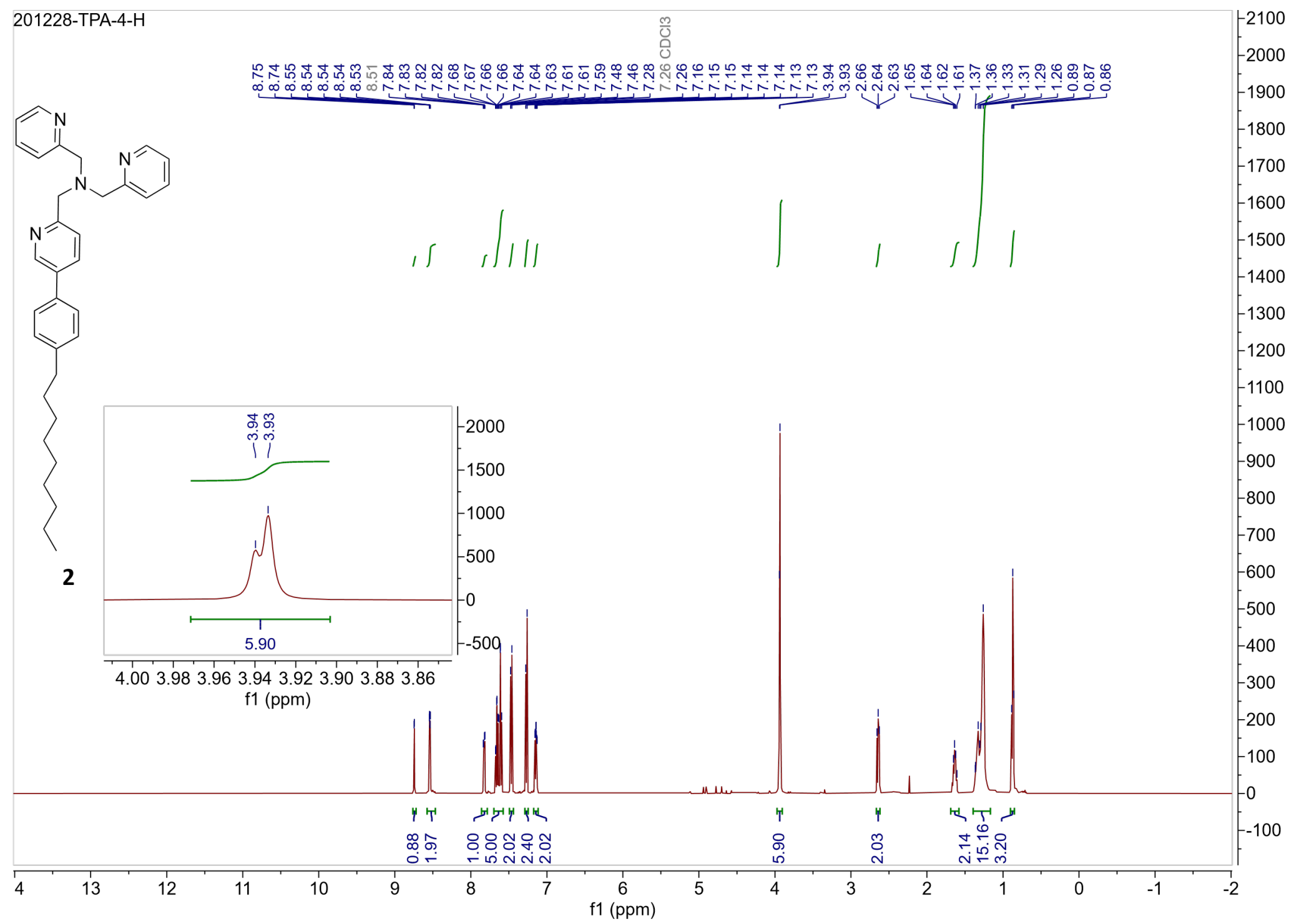


${ }^{13} \mathrm{C}$ NMR

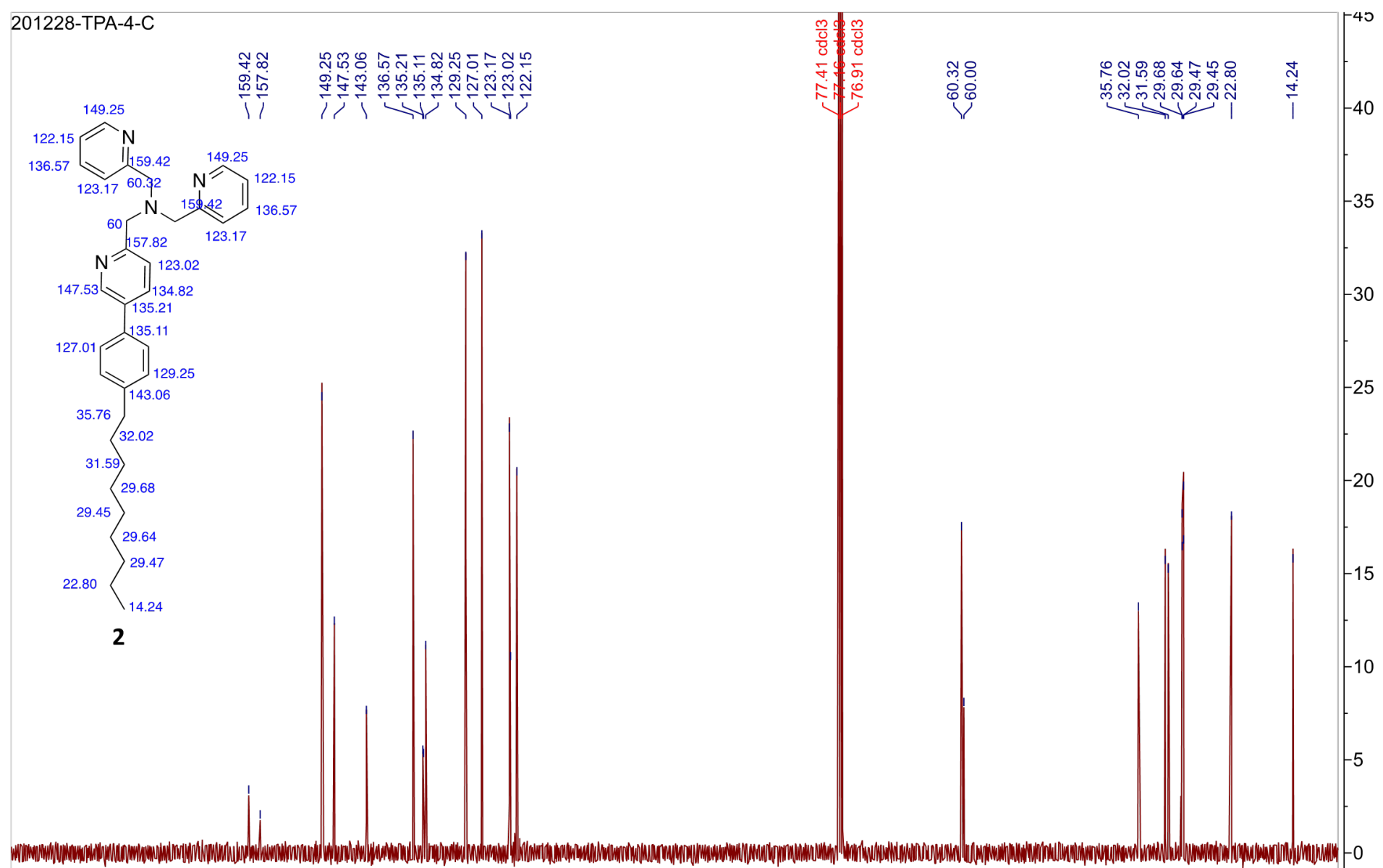

$\begin{array}{llllll}190 & 180 & 170 & 160 & 150 & 140\end{array}$

100
$f 1$

$\begin{array}{llllllllll}1 & 1 & 1 & 1 & 1 & 1 & 1 & 10 & 10 & \end{array}$




\section{ESI-MS}

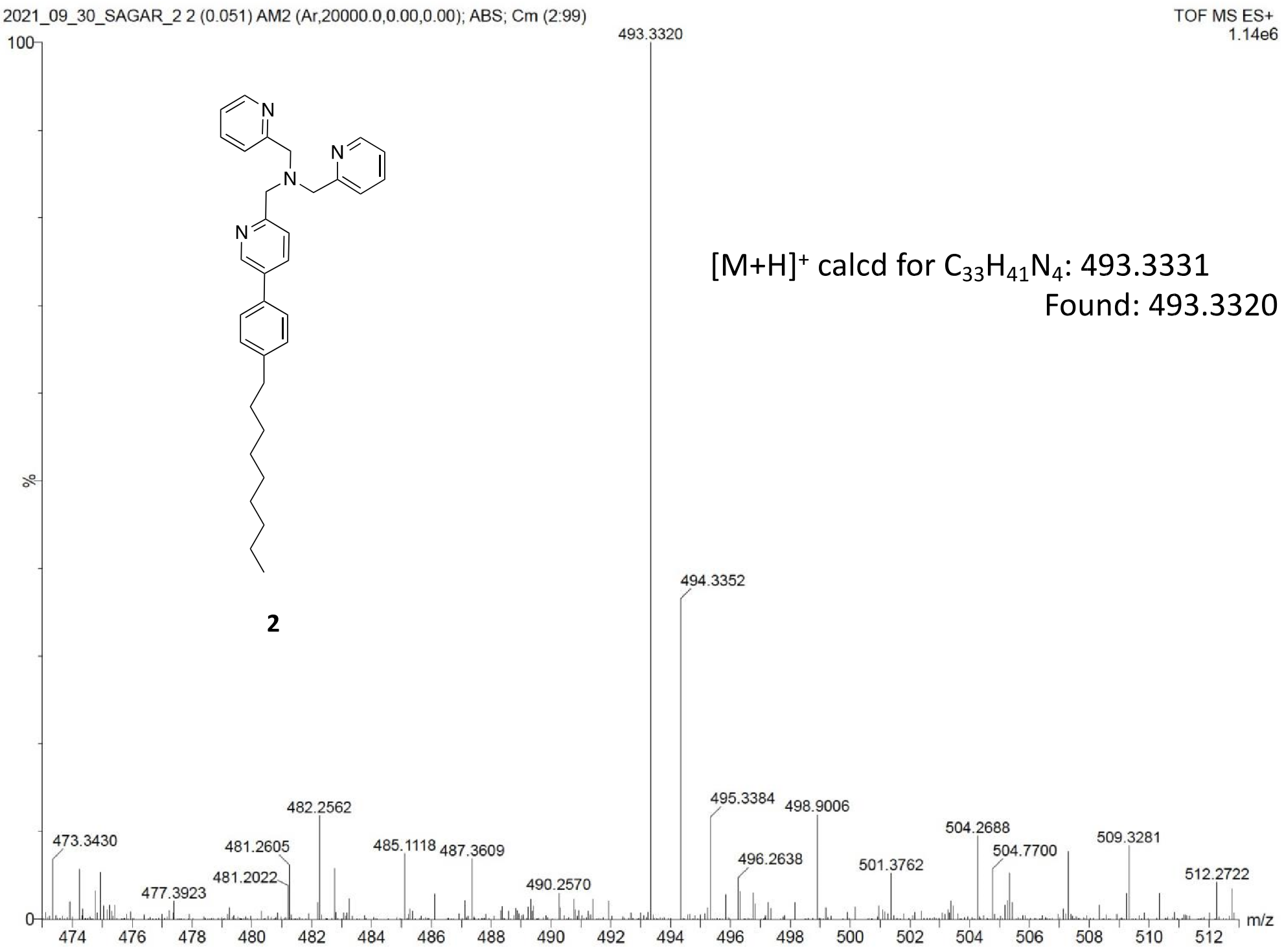




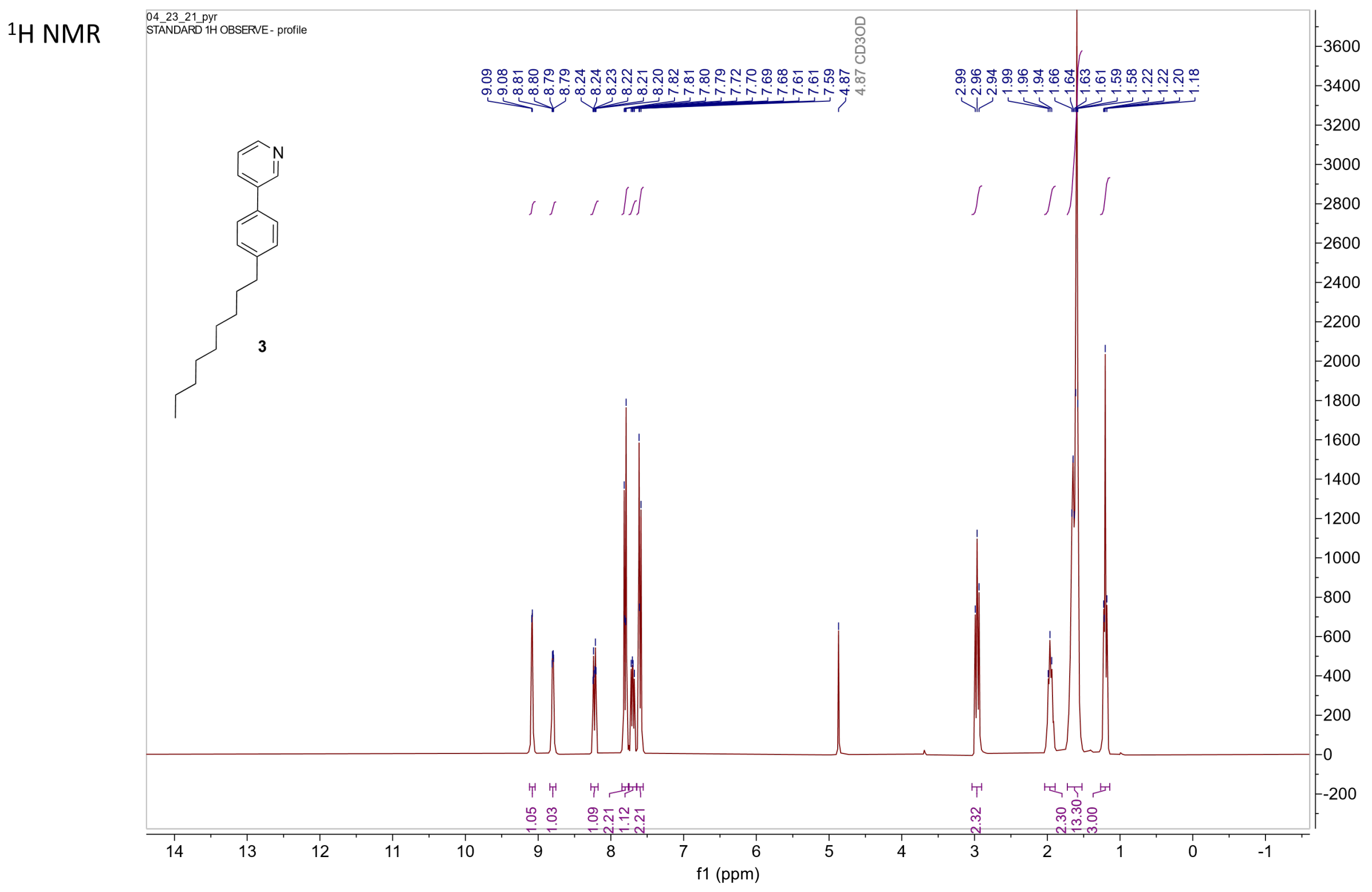




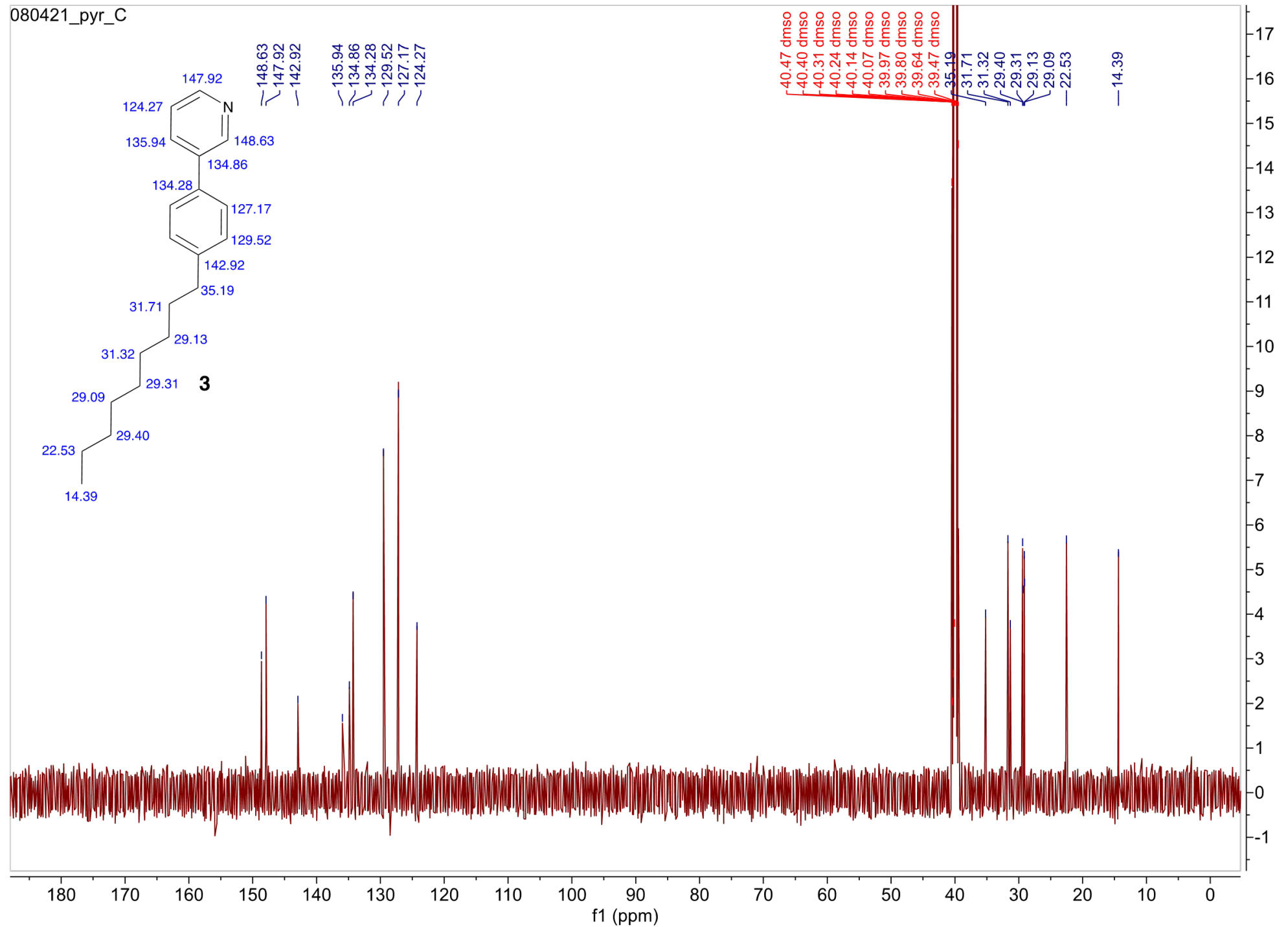




\section{ESI-MS}

2021_08_19_SAGAR_V_PYR_4 49 (0.844) AM2 (Ar,20000.0,0.00,0.00); ABS; Cm (2:117)

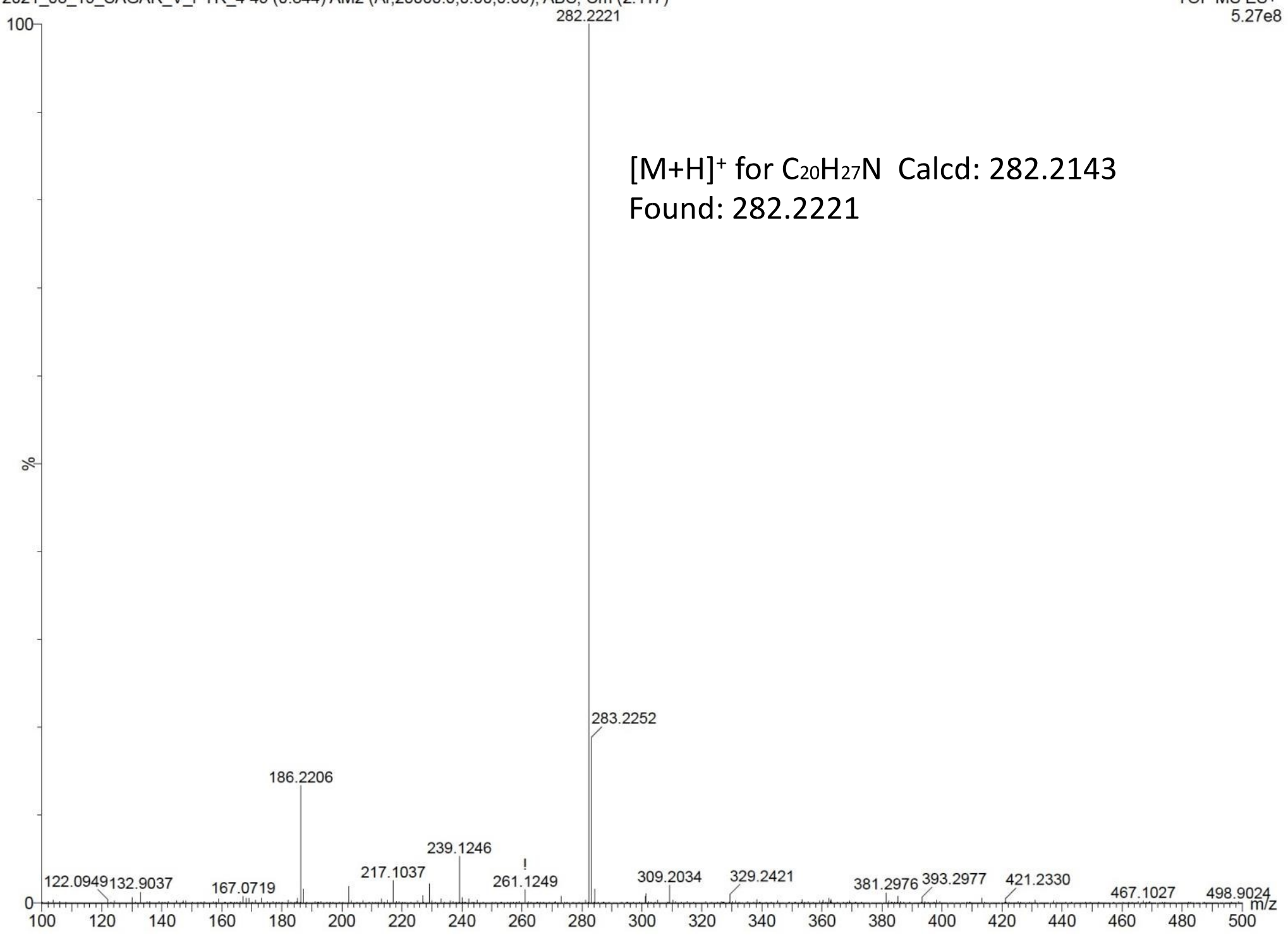

